\title{
Theory and Design of $M$-Channel Maximally Decimated Quadrature Mirror Filters with Arbitrary $M$, Having the Perfect-Reconstruction Property
}

\author{
P. P. VAIDYANATHAN, MEMBER, IEEE
}

\begin{abstract}
Based on the concept of losslessness in digital filter structures, this paper derives a general class of maximally decimated $M$ channel quadrature mirror filter banks that lead to perfect reconstruction. The perfect-reconstruction property guarantees that the reconstructed signal $\hat{x}(n)$ is a delayed version of the input signal $x(n)$, i.e., $\hat{x}(n)=x\left(n-n_{0}\right)$. It is shown that such a property can be satisfied if the alias component matrix ( $\mathrm{AC}$ matrix for short) is unitary on the unit circle of the $z$ plane. The number of channels $M$ is arbitrary, and when $M$ is two, the results reduce to certain recently reported 2-channel perfect-reconstruction QMF structures. A procedure, based on recently reported FIR cascaded-lattice structures, is presented for optimal design of such FIR $M$-channel filter banks. Design examples are included.
\end{abstract}

\section{INTRODUCTION}

Q UADRATURE mirror filter (QMF) banks have received considerable attention during the past several years because of a wide variety of engineering applications [1]-[13]. An $M$-channel QMF bank is shown in Fig. 1 , where $H_{0}(z), H_{1}(z), \cdots, H_{M-1}(z)$ are the transfer functions of analysis bank filters, and $F_{0}(z), F_{1}(z)$, $\cdots, F_{M-1}(z)$ represent the synthesis filters. In the analysis bank, the incoming signal $x(n)$ is split into $M$ frequency bands by filtering, and each subband signal is maximally decimated, i.e., decimated by a factor of $M$. The $M$ decimated signals are then processed in the synthesis bank by interpolating each signal, filtering, and then adding the $M$ filtered signals. In a typical application of such a system, the $M$ decimated signals in the analysis bank are coded and transmitted. The motivation for such signal splitting and coding before transmission is a wellunderstood topic, and is covered well in the literature [1][5].

A common requirement in most applications is that the reconstructed signal $\hat{x}(n)$ should be "as close" to $x(n)$ as possible, in a certain well-defined sense. The reconstructed signal in general suffers from aliasing error because the analysis bank filters $H_{k}(z)$ that precede the decimators are not ideal. In practice, for a given set of analysis filters $H_{k}(z)$, the synthesis filters $F_{k}(z)$ can be

Manuscript received May 19, 1986; revised September 8, 1986. This work was supported in part by the National Science Foundation under Grant ECS 84-04245 and in part by Caltech's Program in Advanced Technology sponsored by Aerojet General, General Motors, GTE, and TRW.

The author is with the Department of Electrical Engineering, California Institute of Technology, Pasadena, CA 91125.

IEEE Log Number.8612887.

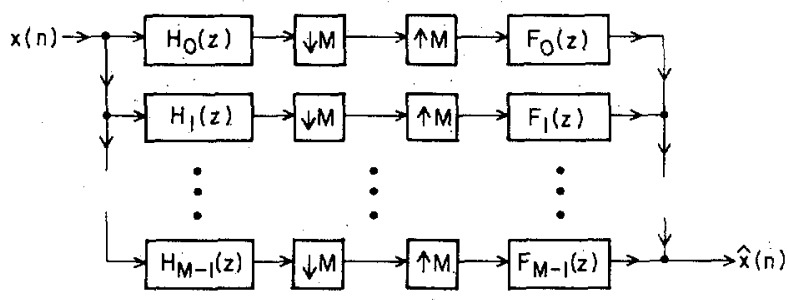

Fig. 1. The $M$-channel maximally decimated parallel QMF bank.

chosen so as to reduce the effect of this aliasing caused by the decimation operation.

It is well known that in two-channel QMF banks (i.e., $M=2$ ) perfect cancellation of aliasing can be accomplished by a simple choice of the functions $F_{0}(z)$ and $F_{1}(z)$ [1]-[5]. For the case of $M$ channels, if $M$ is a power of two, tree structures with two-channel QMF banks can be built that are free from aliasing. For arbitrary $M$, approximate cancellation of aliasing can be accomplished in an elegant manner [8]-[10], whereas perfect cancellation of aliasing can be accomplished with somewhat more complicated synthesis bank filters [11]-[15].

The most general expression for $\hat{X}(z)$ is of the form [3], [7]

$$
\hat{X}(z)=\frac{1}{M} \sum_{l=0}^{M-1} X\left(z W^{-l}\right) \sum_{k=0}^{M-1} H_{k}\left(z W^{-l}\right) F_{k}(z)
$$

where $W=e^{-j 2 \pi / M}$. In (1a), $X\left(z W^{-l}\right), l \neq 0$ represents the aliasing terms. Aliasing is cancelled if and only if the following set of relations holds:

$$
\begin{aligned}
& {\left[\begin{array}{llll}
H_{0}(z) & H_{1}(z) & \cdots & H_{M-1}(z) \\
H_{0}\left(z W^{-1}\right) & H_{1}\left(z W^{-1}\right) & \cdots & H_{M-1}\left(z W^{-1}\right) \\
\vdots & & & \\
H_{0}\left(z W^{-M+1}\right) & H_{1}\left(z W^{-M+1}\right) & \cdots & H_{M-1}\left(z W^{-M+1}\right)
\end{array}\right]} \\
& \cdot\left[\begin{array}{c}
F_{0}(z) \\
F_{1}(z) \\
\vdots \\
F_{M-1}(z)
\end{array}\right]=\left[\begin{array}{l}
T(z) \\
0 \\
\vdots \\
0
\end{array}\right]
\end{aligned}
$$

The above $M \times M$ matrix has been referred to as the alias component matrix (AC matrix) in the literature [7]. Once 
aliasing has been cancelled, the structure of Fig. 1 is time invariant (and of course linear), and $\hat{X}(z)$ is related to $X(z)$ by a transfer function $T(z)$ :

$$
T(z) \triangleq \frac{\hat{X}(z)}{X(z)}=\frac{1}{M} \cdot \sum_{k=0}^{M-1} F_{k}(z) H_{k}(z) .
$$

Thus, $T(z)$ represents the "distortion" caused by the alias-free analysis-synthesis system. An alias-free system is said to have no amplitude distortion if $T(z)$ is a (stable) all-pass function, whereas if $T(z)$ is a linear-phase FIR function; then the system is free from phase distortion. Depending upon the application in hand, it is always possible to choose the set of filters $\left\{F_{k}(z)\right\}$ (for a given set of analysis filters $\left.\left\{H_{k}(z)\right\}\right)$ such that either the amplitude distortion is zero or the phase distortion is zero [11][13].

If an alias-free system is such that the quantity $T(z)$ is a delay, then both amplitude and phase distortions are zero. Such QMF banks are called perfect-reconstruction banks and satisfy

$$
\hat{X}(z)=c z^{-n_{0}} X(z)
$$

for some positive integer $n_{0}$. Here $c$ is an arbitrary constant.

In principle, one can always set $T(z)=z^{-n_{0}}$ in (1b) and invert the AC matrix in order to obtain the synthesis filters $F_{k}(z)$ that would lead to perfect reconstruction. This approach, however, is of little use in practice as it often leads to synthesis filters of very high order which in addition are typically unstable. This motivates us to look at the perfect-reconstruction problem from other points of view that do not involve the inversion of the $\mathrm{AC}$ matrix.

A simple way to obtain perfect reconstruction is to choose the analysis and synthesis filters according to

$$
H_{k}(z)=z^{-k}, \quad F_{k}(z)=z^{-(M-1-k)} .
$$

It is easily verified that such a choice satisfies (3) with $n_{0}$ $=M-1$. (See Appendix A.) However, the filtering functions $H_{k}(z)$ are trivial, and such a structure has little practical value.

A fundamental result has recently been established by Smith and Barnwell [6] who showed that two-channel perfect-reconstruction QMF banks can indeed be constructed, while at the same time accomplishing nontrivial FIR filtering functions $H_{0}(z), H_{1}(z)$. The result is based on an important property satisfied by linear-phase FIR half-band filters, and the designs of $H_{0}(z)$ and $H_{1}(z)$ are based on the spectral-factorization of an appropriately conditioned half-band filter. This problem has also been recently addressed by Mintzer [16].

One of the main aims of our paper here is the extension of these perfect-reconstruction results for the case of $M$ channels, with arbitrary $M$. Referring to the maximally decimated QMF structure of Fig. 1, we show how the transfer functions $\left\{H_{k}(z)\right\}$ and $\left\{F_{k}(z)\right\}$ can be constructed such that aliasing is perfectly cancelled, and in addition (3) is exactly satisfied. Our solution is such that
$H_{k}(z)$ and $F_{k}(z)$ are FIR, for all $k, 0 \leq k \leq M-1$. Moreover, if $N-1$ is the order of each of the filters $H_{k}(z)$, then the order of each of $F_{k}(z)$ is also $N-1$.

The notion of polyphase networks in signal splitting and reconstruction [3], [30], [31] enables one to address many theoretical and practical issues in a unified manner [11][13]. In this paper, we make further use of this tool by defining generalized polyphase structures which can be useful even when the analysis-bank filters are entirely unrelated to each other. The role of all-pass functions and losslessness in signal splitting and reconstruction applications has been noticed and analyzed by some authors in the past [11], [12], [32], [34], thus leading to IIR QMF banks with (no aliasing and) no amplitude distortion. Such IIR QMF banks do lead to phase distortion (because $T(z)$ of (2) is an all-pass function in these examples) which can be compensated by an equalizer. In this paper, we take advantage of the result that multivariable FIR lossless functions can be appropriately employed in a QMF bank in order to reduce all types of distortion to zero, thereby resulting in perfect reconstruction.

Certain simple solutions to the perfect-reconstruction problem, which are of restricted use, have recently been presented [11]-[13] and do not in general have FIR components. ${ }^{1}$ The question of existence of perfect-reconstruction QMF banks for arbitrary $M$, with FIR analysis and synthesis filters, need not bother us. A simple example of such a system is obtained in Appendix A. More examples can be found in [15]. However, our purpose here is to provide new solutions based on the observation that the concept of losslessness in digital networks [17], [20] is closely related to the concept of signal reconstruction in maximally decimated QMF banks. Our results are such that the FIR filters in the analysis bank have the same length as those in the synthesis bank. In order to render the paper readable in a self-contained manner, we define the notion of losslessness in Section II. We then review the fundamental two-channel perfect-reconstruction results [6] in order to place them in the context of the lossless structural framework. Section III introduces the general $M$-channel perfect-reconstruction circuit. This section derives a set of sufficient conditions for perfect reconstruction with arbitrary $M$. A set of necessary and sufficient conditions is also included in this section.

When $M$ is a power of two, it is well known that tree structures based on the two-channel building blocks in [6] can be used in order to obtain perfect reconstruction. Section IV includes a proof that such Smith-Barnwell tree structures satisfy the set of sufficient conditions developed in Section III. Such a proof is encouraging because it shows that the sufficient conditions we develop are not unduly restrictive, and do not disable us from obtaining good stopband attenuation for the analysis filters.

Since the design of the analysis bank in the two-channel

\footnotetext{
'In a recent conversation with $\mathrm{M}$. Vetterli at the ICASSP' 86 Tokyo Conference, we learned that Dr. Vetterli has made similar observations [14], [15].
} 
case [6], [16] is based on factorization of a half-band filter, a natural attempt in the case of $M$ channels is to try designing the analysis bank based on the factorization of FIR $M$ th band filters [21]. Surprisingly, this attempt does not work for all $M$ (but works only for restricted $M$, viz. $M=2$ ), i.e., the resulting analysis filters do not have good frequency response. The theoretical reason for this is explained in Section V. We feel that it is useful to be aware of this result.

In Section VI we develop a class of FIR lattice structures for the analysis and synthesis banks based on some recent work [18]-[20] on FIR lattice filters. The purpose is to enable us to set up an optimization algorithm that designs $H_{k}(z)$ so as to have good stopband attenuation. The lattice structures are such that they automatically satisfy the set of sufficient conditions required for perfect reconstruction. Accordingly, if we optimize the parameters of this lattice, it is equivalent to finding the best set of transfer functions $\left\{H_{k}(z)\right\}$ by searching only among the class of transfer functions that already satisfy conditions for alias-free perfect reconstruction. We feel that this is a major advantage of using the lattice structures in the optimization. A design example is included here. Finally, Section VII examines recursive QMF banks in the context of lossless matrices.

\section{A. Notations Used in the Paper}

Superscript $T$ stands for matrix (or vector) transposition, whereas superscript dagger $(\dagger)$ stands for transposition followed by complex conjugation. Boldface italic letters indicate matrices and vectors. Superscript asterisk $(*)$ stands for complex conjugation, while subscript asterisk denotes conjugation of coefficients of the function or matrix. The tilde accent on a function $\boldsymbol{F}(z)$ is defined such that, on the unit circle, $\tilde{\boldsymbol{F}}(z)=\boldsymbol{F}^{\dagger}(z)$. Thus, for arbitrary $z, \tilde{\boldsymbol{F}}(z)=\boldsymbol{F}_{*}^{T}\left(z^{-1}\right)$, and for functions with real coefficients, $\tilde{\boldsymbol{F}}(z)=\boldsymbol{F}^{T}\left(z^{-1}\right)$.

Since our work was primarily motivated by the original contributions in [6], these results are often referenced in this paper. For ease of reference, the perfect-reconstruction two-channel structure in [6] will be called the SmithBarnwell structure (and abbreviated as the SB structure).

\section{The Smith-Barnwell QMF Bank in the Context of Lossless Structures}

A single-input single-output digital transfer function $G(z)$ is said to be lossless [17] if it is stable and satisfies $\left|G\left(e^{j \omega}\right)\right|=1$ for all $\omega$. Such a function is merely an allpass function. An $m$-input $p$-output transfer function (i.e., a general matrix transfer function) $T(z)$ is said to be lossless if it is stable and satisfies $\boldsymbol{T}^{\dagger}\left(e^{j \omega}\right) \boldsymbol{T}\left(e^{j \omega}\right)=\boldsymbol{I}$ for all $\omega$ or equivalently, by analytic continuation,

$$
\tilde{\boldsymbol{T}}(z) \boldsymbol{T}(z)=\boldsymbol{T}(z) \tilde{\boldsymbol{T}}(z)=\boldsymbol{I}, \quad \text { for all } z .
$$

In essence, a lossless function or matrix is stable and is unitary on the unit circle. Such a $T(z)$ can be looked upon as a multiinput, multioutput all-pass function. These concepts are discrete-time versions of the notions of lossless-

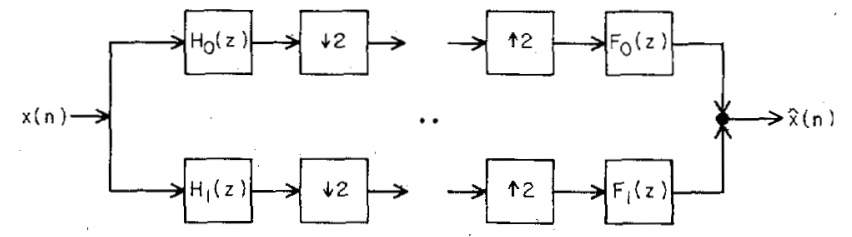

Fig. 2. The two-channel QMF bank.

ness in continuous-time passive network theory [22], [23] and have been used in the past in a completely different context, viz., for low-sensitivity digital filter design [17], [18], [24].

If $G(z)$ is FIR and lossless, then it has to be a pure delay, i.e., $G(z)=z^{-\alpha}, \alpha=$ integer. However, it is possible to have matrix-valued FIR functions $T(z)$ that are more complicated than a delay. For example,

$$
\boldsymbol{T}(z)=\frac{\left[\begin{array}{ll}
1+z^{-1} & 1-z^{-1} \\
1-z^{-1} & 1+z^{-1}
\end{array}\right]}{2}
$$

is easily verified to be lossless, as it satisfies (5).

\section{A. Revisiting the Perfect-Reconstruction Structure}

Now consider the two-channel QMF structure of Fig. 2. Here $H_{0}(z)$ and $H_{1}(z)$ are low-pass and high-pass transfer functions, respectively. The signal $\hat{X}(z)$ is given by

$$
\begin{aligned}
\hat{X}(z)= & {\left[H_{0}(z) F_{0}(z)+H_{1}(z) F_{1}(z)\right] X(z) / 2 } \\
& +\left[H_{0}(-z) F_{0}(z)+H_{1}(-z) F_{1}(z)\right] X(-z) / 2 .
\end{aligned}
$$

The term involving $X(-z)$ is the aliasing term and is required to be made equal to zeto. In the scheme due to Smith and Barnwell [6], the following relation between the transfer functions is enforced: ${ }^{2}$

$$
\begin{aligned}
& H_{1}(z)=z^{-(N-1)} H_{0}\left(-z^{-1}\right) \\
& F_{0}(z)=z^{-(N-1)} H_{0}\left(z^{-1}\right) \\
& F_{1}(z)=z^{-(N-1)} H_{1}\left(z^{-1}\right)
\end{aligned}
$$

where $N-1$ is the order of $H_{0}(z)$. Here $H_{0}(z)$ and $H_{1}(z)$ are constrained to satisfy

$$
H_{0}\left(z^{-1}\right) H_{0}(z)+H_{1}\left(z^{-1}\right) H_{1}(z)=1, \quad \text { for all } z \text {. }
$$

Without loss of generality, $N-1$ can be assumed to be odd. (See Section V.) The conditions (7)-(9) are sufficient to enforce the following condition:

$$
H_{0}(-z) F_{0}(z)+H_{1}(-z) F_{1}(z)=0
$$

and thereby cancel aliasing. The relation (6) then becomes

\footnotetext{
${ }^{2}$ The relations (7)-(9) do not appear to be the same as those in [6] because we have displayed a causal version of those equations.
} 


$$
\begin{aligned}
\hat{X}(z)= & \frac{1}{2} z^{-(N-1)}\left[H_{0}\left(z^{-1}\right) H_{0}(z)\right. \\
& \left.+H_{1}\left(z^{-1}\right) H_{1}(z)\right] X(z)
\end{aligned}
$$

which in view of (10) reduces to (3). In order to ensure that condition (10) indeed holds, a design procedure is proposed in [6] based on the spectral factorization of a linear-phase FIR half-band filter with positive amplitude response.

The AC matrix for a two-channel QMF bank is

$$
\boldsymbol{H}(z)=\left[\begin{array}{ll}
H_{0}(z) & H_{1}(z) \\
H_{0}(-z) & H_{1}(-z)
\end{array}\right] .
$$

It can be verified that condition (7) implies that the columns of (13) are "orthogonal," that is,

$$
H_{0}\left(z^{-1}\right) H_{1}(z)+H_{0}\left(-z^{-1}\right) H_{1}(-z)=0 .
$$

Moreover, each column of (13) satisfies

$$
H_{k}\left(z^{-1}\right) H_{k}(z)+H_{k}\left(-z^{-1}\right) H_{k}(-z)=1, \quad k=0,1
$$

as can be verified by employing (7) and (10). Properties (14) and (15) are precisely equivalent to the statement that $\boldsymbol{H}(z)$ is lossless, i.e., satisfies the condition $\tilde{\boldsymbol{H}}(z) \boldsymbol{H}(z)$ $=1$.

In contrast, consider the more standard two-channel QMF bank [1]-[5]. For such a filter bank, the transfer function $H_{0}(z)$ is restricted to be a linear-phase FIR filter of odd order $N-1$, and the remaining transfer functions are given by

$$
\begin{aligned}
& H_{1}(z)=H_{0}(-z), F_{0}(z)=H_{0}(z), \\
& F_{1}(z)=-H_{1}(z) .
\end{aligned}
$$

With such a choice of transfer functions, aliasing is cancelled and we have [5]

$$
\hat{X}(z)=\left[H_{0}^{2}(z)-H_{0}^{2}(-z)\right] X(z) / 2 .
$$

It can furthermore be shown that $\boldsymbol{H}(z)$ satisfies

$$
\tilde{\boldsymbol{H}}(z) \boldsymbol{H}(z)=\left[H_{0}\left(z^{-1}\right) H_{0}(z)+H_{1}\left(z^{-1}\right) H_{1}(z)\right] \cdot \boldsymbol{I}
$$

which reduces to

$$
\tilde{\boldsymbol{H}}(z) \boldsymbol{H}(z)=z^{(N-1)}\left[H_{0}^{2}(z)-H_{0}^{2}(-z)\right] \cdot \boldsymbol{I},
$$

because of (16) and the fact that $H_{0}(z)$ is an odd-order filter with symmetric impulse response. In order for $\boldsymbol{H}(z)$ to be lossless, it is again necessary to satisfy (10). But it is well known that two linear-phase FIR transfer functions $H_{0}(z)$ and $H_{1}(z)$ cannot satisfy $(10)$ unless they are trivial combinations of delays [29].

In summary, the AC matrix in the Smith-Barnwell perfect-reconstruction structure is lossless, whereas the AC matrix in the standard two-channel alias-free structures is not $^{3}$ except in trivial situations.

\footnotetext{
${ }^{3}$ In this paper, the term "standard two-channel QMF bank" stands for a structure as in Fig. 2, with the transfer functions satisfying (16) and with $H_{0}(z)$ representing an odd order $(=N-1)$ linear-phase FIR filter, with symmetric impulse response.
}

\section{B. Revisiting Polyphase Implementations of Two- Channel Structures}

Let the transfer functions $H_{0}(z)$ and $H_{1}(z)$ be written in the form

$$
\begin{aligned}
& H_{0}(z)=E_{00}\left(z^{2}\right)+z^{-1} E_{01}\left(z^{2}\right) \\
& H_{1}(z)=E_{10}\left(z^{2}\right)+z^{-1} E_{11}\left(z^{2}\right) .
\end{aligned}
$$

Clearly such a representation is always possible. We therefore have

$$
\left[\begin{array}{ll}
H_{0}(z) & H_{0}(-z) \\
H_{1}(z) & H_{1}(-z)
\end{array}\right]=\left[\begin{array}{ll}
E_{00}\left(z^{2}\right) & E_{01}\left(z^{2}\right) \\
E_{10}\left(z^{2}\right) & E_{11}\left(z^{2}\right)
\end{array}\right]\left[\begin{array}{cc}
1 & 1 \\
z^{-1} & -z^{-1}
\end{array}\right] .
$$

Defining the $2 \times 2$ matrix $E(z)=\left[E_{k l}(z)\right], 0 \leq k, l \leq$ 1 , we obtain from (22)

$$
\boldsymbol{H}(z)=\left[\begin{array}{rr}
1 & z^{-1} \\
1 & -z^{-1}
\end{array}\right] \boldsymbol{E}^{T}\left(z^{2}\right)
$$

Thus,

$$
\tilde{\boldsymbol{H}}(z) \boldsymbol{H}(z)=2 \boldsymbol{E}\left(z^{-2}\right) \boldsymbol{E}^{T}\left(z^{2}\right)
$$

which shows that $\boldsymbol{H}(z)$ is lossless if and only if $\sqrt{2} \boldsymbol{E}(z)$ is lossless. In view of our earlier conclusions, it thus follows that $\sqrt{2} \boldsymbol{E}(z)$ is lossless for the Smith-Barnwell scheme, whereas it is not for the standard QMF scheme. For the standard QMF structure it can be verified that $E_{k l}(z)$ satisfy

$$
\begin{aligned}
& E_{10}(z)=E_{00}(z), \quad E_{01}(z)=z^{-m_{1}} E_{00}\left(z^{-1}\right), \\
& E_{11}(z)=-z^{-m_{1}} E_{00}\left(z^{-1}\right)
\end{aligned}
$$

leading to the result

$$
\tilde{E}(z) \boldsymbol{E}(z)=\boldsymbol{E}(z) \tilde{\boldsymbol{E}}(z)=2 E_{00}\left(z^{-1}\right) E_{00}(z) \cdot \boldsymbol{I} \text {. }
$$

In order to develop a physical feeling for these properties, let us redraw Fig. 2 in terms of the $\boldsymbol{E}\left(z^{2}\right)$ matrix. For the SB structure,

$$
\left[\begin{array}{l}
F_{0}(z) \\
F_{1}(z)
\end{array}\right]=z^{-(N-1)}\left[\begin{array}{l}
H_{0}\left(z^{-1}\right) \\
H_{1}\left(z^{-1}\right)
\end{array}\right]=z^{-(N-1)} E\left(z^{-2}\right)\left[\begin{array}{l}
1 \\
z
\end{array}\right]
$$

whence

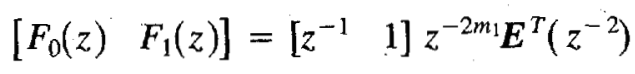

where $m_{1}=(N-2) / 2$. Thus, Fig. 2 can be redrawn as in Fig. 3. For the standard QMF structure also, it can be verified, based on the relations (25), that Fig. 2 can be redrawn as in Fig. 3. Based on standard identities for multirate systems [3], Fig. 3 can in turn be redrawn as in Fig. 4. For the SB structure, since $\sqrt{2} \boldsymbol{E}(z)$ is lossless, the structure of Fig. 2 is therefore eventually equivalent to Fig. 5(a), whereas for the standard QMF structure, it is equivalent to Fig. 5(b), which clearly shows us that the standard structure is alias free (see Appendix A), while the SB structure, in addition to being alias free, has the perfect-reconstruction property. From Fig. 5 it follows 


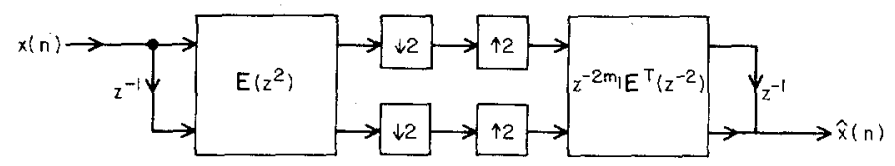

Fig. 3. An equivalent structure for Fig. 2 .

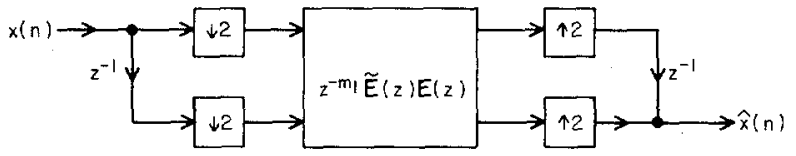

Fig. 4. A redrawing of Fig. 3.

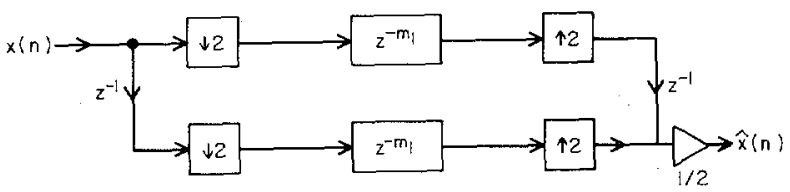

(a)

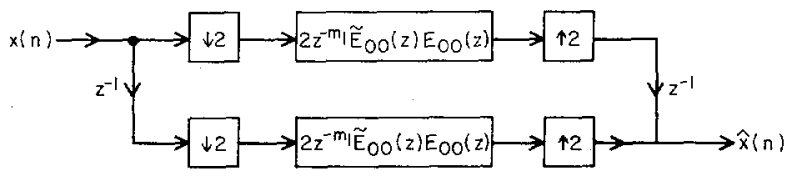

(b)

Fig. 5. The simplified equivalent structures (a) for the SB QMF bank, and (b) for the standard QMF bank.

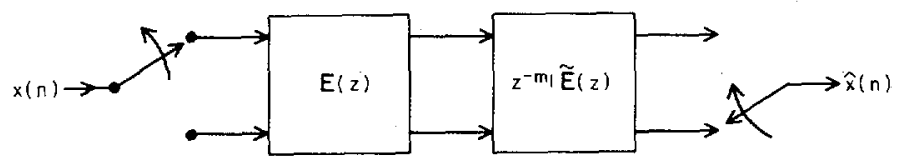

Fig. 6. "Polyphase" implementation of Fig. 3.

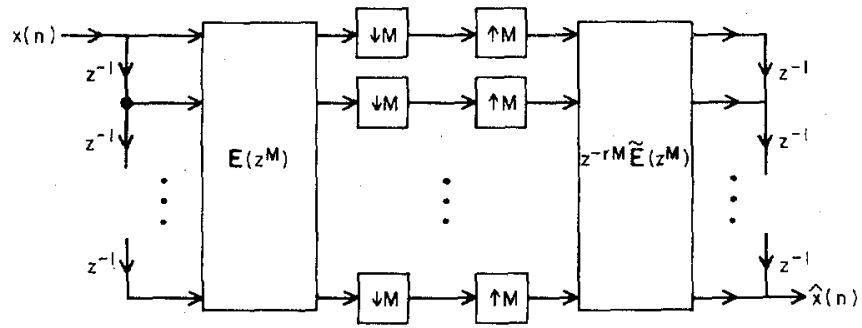

Fig. 7. Extension to $M$-channel QMF banks.

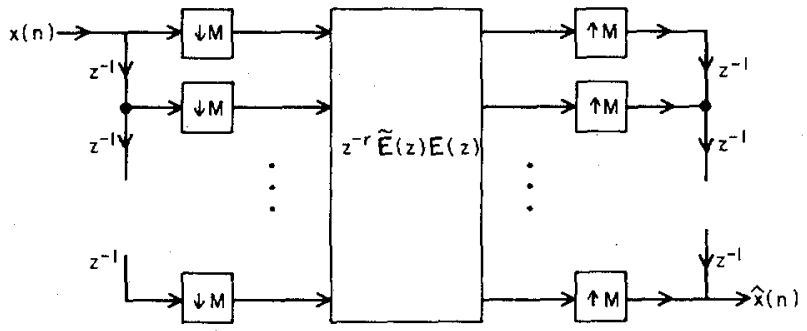

(a)

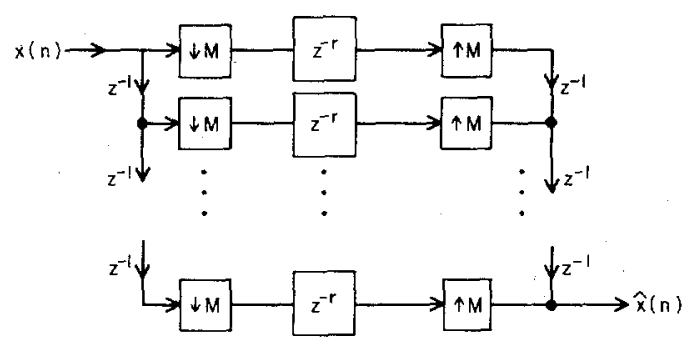

(b)

Fig. 8. (a) A structure that is equivalent to Fig. 7. (b) The final equivalent version of Fig. 7.

(Appendix A) that

$$
\hat{X}(z)= \begin{cases}\frac{1}{2} z^{-(N-1)} X(z) & \text { (SB structure) } \\ 2 z^{-(N-1)} E_{00}\left(z^{-2}\right) E_{00}\left(z^{2}\right) X(z)=2 z^{-1} E_{00}\left(z^{2}\right) E_{01}\left(z^{2}\right) X(z) & \text { (standard structure) }\end{cases}
$$

which are, of course, well-known results.

Notice finally that a "polyphase" implementation results by redrawing Fig. 3 as in Fig. 6. This structure differs from the more well-known polyphase structures [3] (which have diagonal $\boldsymbol{E}(z)$ matrices).

\section{Maximally Decimated $M$-Channel Perfect- Reconstruction Structures}

The interpretation of the two-channel SB structure as in Fig. 3 immediately tells us how the idea can be extended for $M$ channels. Thus, consider Fig. 7 where $\tilde{E}\left(z^{M}\right)$ stands for $\boldsymbol{E}^{T}\left(z^{-M}\right)$ (and more generally stands for $\boldsymbol{E}_{*}^{T}\left(z^{-M}\right)$ if the coefficients in $\boldsymbol{E}(z)$ can be complex). In Fig. 7; $r$ is an integer large enough so that $z^{-r} \tilde{E}(z)$ has no positive powers of $z$. Fig. 7 is clearly equivalent to Fig. 8(a). If $E(z)$ is lossless, this in turn is equivalent to Fig. 8(b). For Fig. 8(b) we can easily show that

$$
\hat{X}(z)=z^{-(M-1+r M)} X(z)
$$

which implies perfect reconstruction. Notice that the analysis filters and synthesis filters are given by

$$
\begin{gathered}
\boldsymbol{h}(z)=\left[\begin{array}{c}
H_{0}(z) \\
H_{1}(z) \\
\vdots \\
H_{M-1}(z)
\end{array}\right]=\boldsymbol{E}\left(z^{M}\right)\left[\begin{array}{l}
1 \\
z^{-1} \\
\vdots \\
z^{-(M-1)}
\end{array}\right] \\
\boldsymbol{f}(z)=\left[\begin{array}{c}
F_{0}(z) \\
F_{1}(z) \\
\vdots \\
F_{M-1}(z)
\end{array}\right]=z^{-r M} \boldsymbol{E}\left(z^{-M}\right)\left[\begin{array}{l}
z^{-(M-1)} \\
z^{-(M-2)} \\
\vdots \\
z^{-1} \\
1
\end{array}\right]
\end{gathered}
$$

Thus, we can state the following result. 


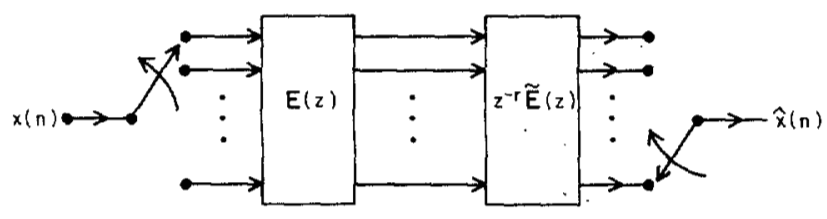

Fig. 9. The polyphase implementation of Fig. 7.

Lemma 3.1: Let $H_{0}(z), H_{1}(z), \cdots, H_{M-1}(z)$ be a set of analysis filters in the maximally decimated structure of Fig. 1. Define $E_{k l}(z)$ for $0 \leq k, l \leq M-1$ by

$$
H_{k}(z)=\sum_{l=0}^{M-1} z^{-l} E_{k l}\left(z^{M}\right) \text {. }
$$

If the matrix

$$
\boldsymbol{E}(z) \triangleq\left[E_{k l}(z)\right]
$$

is lossless, then the set of synthesis filters $\left\{F_{k}(z)\right\}$ defined according to (31b) leads to perfect reconstruction.

Notice that the orders of the synthesis-bank filters and analysis-bank filters are the same. Notice also that if $H_{k}(z)$ are IIR, then there does not exist finite $r$ such that $z^{-r} \tilde{E}(z)$ is causal and stable, hence, the above result is meant to be used for FIR QMF banks alone. Finally, notice that a polyphase implementation of Fig. 7 can immediately be drawn as shown in Fig. 9. Once again, this differs from standard polyphase structures [3] that have diagonal $\boldsymbol{E}(z)$.

The most important practical question is now the following: how do we construct a set of $M$ FIR transfer functions $H_{0}(z), H_{1}(z), \cdots, H_{M-1}(z)$ such that the FIR matrix $\boldsymbol{E}(z)$ defined above is lossless? A trivial solution, of course, is to take $H_{k}(z)$ as in (4) which makes $\boldsymbol{E}(z)=\boldsymbol{I}$ which is clearly lossless. We would like to obtain nontrivial solutions whereby $H_{k}(z)$ are "good" band-pass filters. Fortunately, such a design scheme is rendered feasible because of certain recently reported FIR lattice structures [18]-[20], and is the topic of Section VI.

For the rest of this section, we study certain properties of QMF banks constructed based on the lossless property. The first one pertains to the $\mathrm{AC}$ matrix $\boldsymbol{H}(z)$ given by

$\boldsymbol{H}(z)=$

$$
\left[\begin{array}{llll}
H_{0}(z) & H_{1}(z) & \cdots & H_{M-1}(z) \\
H_{0}\left(z W^{-1}\right) & H_{1}\left(z W^{-1}\right) & \cdots & H_{M-1}\left(z W^{-1}\right) \\
\vdots & & & \\
H_{0}\left(z W^{-M+1}\right) & H_{1}\left(z W^{-M+1}\right) & \cdots & H_{M-1}\left(z W^{-M+1}\right)
\end{array}\right] .
$$

From the definition of $E_{k l}(z)$ as in (32), it follows that

$$
\begin{gathered}
{[\boldsymbol{H}(z)]_{s, k}=H_{k}\left(z W^{-s}\right)=\sum_{l=0}^{M-1} z^{-l} W^{l s} E_{k l}\left(z^{M}\right),} \\
0 \leq s, k \leq M-1
\end{gathered}
$$

whence $\boldsymbol{H}(z)$ can be written as

$$
\boldsymbol{H}(z)=\boldsymbol{W A}(z) \boldsymbol{E}^{T}\left(z^{M}\right)
$$

where $W$ is the $M \times M$ DFT matrix, and $A(z)$ is a diagonal matrix defined as

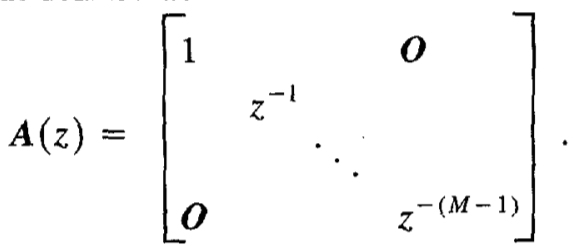

This implies

$$
\tilde{\boldsymbol{H}}(z) \boldsymbol{H}(z)=\boldsymbol{M E}\left(z^{-M}\right) \boldsymbol{E}^{T}\left(z^{M}\right)
$$

establishing the following property.

Property 3.1: $\boldsymbol{H}(z)$ is lossless if and only if $\sqrt{M} \boldsymbol{E}(z)$ is lossless.

The second property pertains to the relation between $\left\{H_{k}(z)\right\}$ and $\left\{F_{k}(z)\right\}$ of any perfect-reconstruction QMF bank. Recall that perfect reconstruction implies

$$
\begin{gathered}
{\left[\begin{array}{llll}
H_{0}(z) & H_{1}(z) & \cdots & H_{M-1}(z) \\
H_{0}\left(z W^{-1}\right) & H_{1}\left(z W^{-1}\right) & \cdots & H_{M-1}\left(z W^{-1}\right) \\
\vdots & & & \\
H_{0}\left(z W^{-M+1}\right) & H_{1}\left(z W^{-M+1}\right) & \cdots & H_{M-1}\left(z W^{-M+1}\right)
\end{array}\right]=\left[\begin{array}{c}
c z^{-n_{0}} \\
0 \\
0 \\
\vdots \\
F_{0}(z) \\
F_{1}(z) \\
\vdots \\
F_{M-1}(z)
\end{array}\right]=\left[\begin{array}{l}
\end{array}\right]}
\end{gathered}
$$

for all $z$. Thus, if $z$ is replaced with $z W^{-1}$ in (38), it continues to hold:

$$
\left.\begin{array}{l}
{\left[\begin{array}{cccc}
H_{0}\left(z W^{-1}\right) & H_{1}\left(z W^{-1}\right) & \cdots & H_{M-1}\left(z W^{-1}\right) \\
H_{0}\left(z W^{-2}\right) & H_{1}\left(z W^{-2}\right) & \cdots & H_{M-1}\left(z W^{-2}\right) \\
\vdots & & & \\
H_{0}(z) & H_{1}(z) & \cdots & H_{M-1}(z)
\end{array}\right]} \\
\cdot\left[\begin{array}{c}
F_{0}\left(z W^{-1}\right) \\
F_{1}\left(z W^{-1}\right) \\
\vdots \\
F_{M-1}\left(z W^{-1}\right)
\end{array}\right]=\left[\begin{array}{c}
c z^{-n_{0}} W^{n_{0}} \\
0 \\
0 \\
\vdots
\end{array}\right]
\end{array}\right]
$$

which can be rewritten as

$$
\begin{aligned}
& {\left[\begin{array}{llll}
H_{0}(z) & H_{1}(z) & \cdots & H_{M-1}(z) \\
H_{0}\left(z W^{-1}\right) & H_{1}\left(z W^{-1}\right) & \cdots & H_{M-1}\left(z W^{-1}\right) \\
\vdots & & \\
H_{0}\left(z W^{-M+1}\right) & H_{1}\left(z W^{-M+1}\right) & \cdots & H_{M-1}\left(z W^{-M+1}\right)
\end{array}\right]} \\
& \quad\left[\begin{array}{c}
F_{0}\left(z W^{-1}\right) \\
F_{1}\left(z W^{-1}\right) \\
\vdots \\
F_{M-1}\left(z W^{-1}\right)
\end{array}\right]=\left[\begin{array}{ll}
0 & \\
c z^{-n_{0}} W^{n 0} \\
0 \\
\vdots \\
0
\end{array}\right] .
\end{aligned}
$$


In general, by replacing $z$ with $z W^{-k}, 0 \leq k \leq M-1$ in (38), we arrive at a set of $M$ matrix-vector equations of the form in (40). These can be put together into a compact equation:

$$
\boldsymbol{H}(z) \boldsymbol{F}(z)=c z^{-n_{0}}\left[\begin{array}{rrrr}
1 & & & \\
& W^{n 0} & & \\
& & W^{2 n_{0}} & \\
& \boldsymbol{O} & \ddots & \\
& & & W^{(M-1) n_{0}}
\end{array}\right]
$$

where $\boldsymbol{F}(z)$ is defined by

$$
\begin{aligned}
& \boldsymbol{F}(z) \\
& \quad=\left[\begin{array}{cccc}
F_{0}(z) & F_{0}\left(z W^{-1}\right) & \cdots & F_{0}\left(z W^{-M+1}\right) \\
F_{1}(z) & F_{1}\left(z W^{-1}\right) & \cdots & F_{1}\left(z W^{-M+1}\right) \\
\vdots & \vdots & \vdots & \vdots \\
F_{M-1}(z) & F_{M-1}\left(z W^{-1}\right) & \vdots & F_{M-1}\left(z W^{-M+1}\right)
\end{array}\right] .
\end{aligned}
$$

The above argument therefore establishes the following result.

Property 3.2: The maximally decimated $M$-channel QMF structure of Fig. 1 gives rise to perfect reconstruction if and only if (41) holds, where $\boldsymbol{H}(z)$ is the AC matrix and $F(z)$ is defined as in (42).

This property gives rise to an important corollary. Thus, let $\boldsymbol{H}(z)$ be lossless. Then

$$
\boldsymbol{H}(z) \boldsymbol{H}_{*}^{T}\left(z^{-1}\right)=\boldsymbol{I}
$$

If, in addition, $F_{k}(z)$ are such that there is perfect reconstruction, then (41) holds; hence,

$$
\boldsymbol{F}(z)=c z^{-n_{0}} \boldsymbol{H}_{*}^{T}\left(z^{-1}\right)\left[\begin{array}{ccc}
1 & & 0 \\
& W^{n_{0}} & \\
& \ddots & \\
O & & W^{(M-1) n_{0}}
\end{array}\right]
$$

which shows that $F_{k}(z)$ must be of the form

$$
F_{k}(z)=c z^{-n_{0}} H_{k}\left(z^{-1}\right) \text {. }
$$

In fact, we can state the following.

Property 3.3: Let the AC matrix $\boldsymbol{H}(z)$ be lossless. Then the structure of Fig. 1 gives rise to perfect reconstruction if and only if $F_{k}(z)$ are related to $H_{k}(z)$ as in (45) where $c$ is an arbitrary constant.

Notice, as a verification, that for the case of $M=2$, the SB structure satisfies property 3.3. The importance of this property is that, if we know how to choose $H_{k}(z)$ such that $\boldsymbol{H}(z)$ is lossless, then there exists a unique way to choose $F_{k}(z)$ so that the reconstruction is perfect. Notice also that if $\boldsymbol{H}(z)$ is lossless but $H_{k}(z)$ not FIR, then $F_{k}(z)$ in (45) are unstable (assuming $H_{k}(z)$ are stable, of course), and hence, there does not exist a stable perfectreconstruction technique. Thus, for IIR filters, the strategy would be to avoid forcing $\boldsymbol{H}(z)$ to be lossless. Fi-

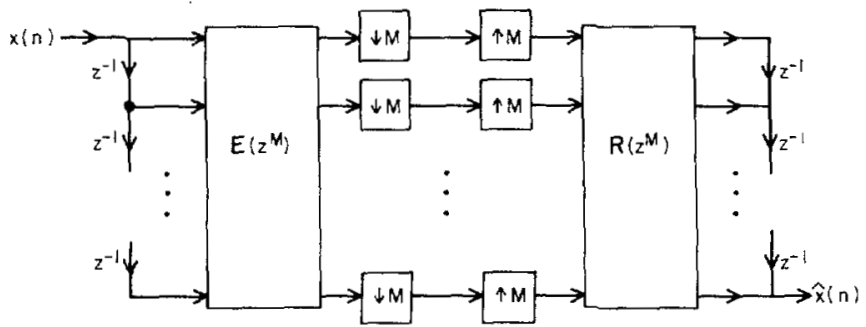

(a)

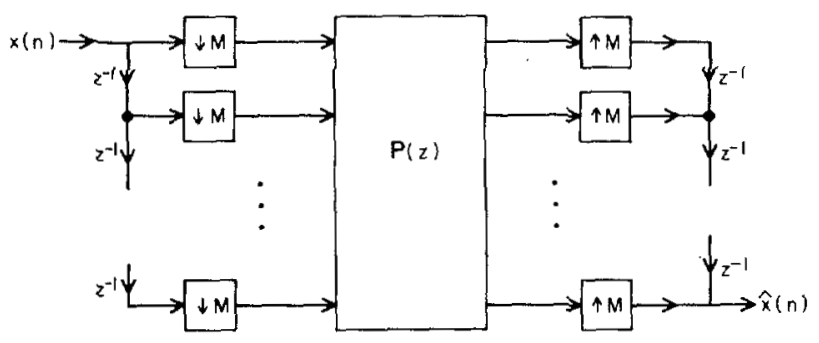

(b)

Fig. 10. Pertaining to lemma 3.2

nally, it should be emphasized that (45) is a necessary condition for perfect reconstruction only if $\boldsymbol{H}(z)$ is lossless.

Next, let us assume that Fig. 1 performs perfect reconstruction so that (41) holds. If in addition (45) holds, then by direct substitution into (41) we can verify that

$$
\boldsymbol{H}(z) \boldsymbol{H}_{*}^{T}\left(z^{-1}\right)=\boldsymbol{I} \text {. }
$$

In other words, we have the following.

Property 3.4: Let the structure of Fig. 1 be such that it performs perfect reconstruction. Furthermore, let $F_{k}(z)$ be related to $H_{k}(z)$ as in (45). Then $\boldsymbol{H}(z)$ is necessarily lossless.

The above properties are essentially various useful manifestations of certain sets of sufficient conditions for perfect reconstruction. A natural question that arises in this context is: what is a set of necessary and sufficient conditions? We now turn our attention to this issue.

Given an arbitrary set of filters $\left\{H_{k}(z)\right\}$ and $\left\{F_{k}(z)\right\}$, we can also express $H_{k}(z)$ in the form (32) and $F_{k}(z)$ in the form

$$
F_{k}(z)=\sum_{l=0}^{M-1} z^{-(M-1-l)} R_{l k}\left(z^{M}\right)
$$

Letting

$$
\boldsymbol{R}(z) \triangleq\left[\boldsymbol{R}_{l k}(z)\right]
$$

Fig. 1 can therefore be redrawn as in Fig. 10(a). Letting $\boldsymbol{P}(z)=\boldsymbol{R}(z) \boldsymbol{E}(z)$, Fig. 10(a) can be redrawn as in Fig. $10(b)$. What is a set of necessary and sufficient conditions on $\boldsymbol{P}(z)$ so that (3) holds? In order to answer this question, first note that $\hat{X}(z)$ can be expressed as

$$
\begin{aligned}
\hat{X}(z)= & \frac{z^{-(M-1)}}{M} \sum_{k=0}^{M-1} X\left(z W^{k}\right) \sum_{l=0}^{M-1} W^{-k l} \\
& \cdot \sum_{s=0}^{M-1} z^{-(l-s)} P_{s, l}\left(z^{M}\right)
\end{aligned}
$$


where $P_{s, l}(z)$ is the $(s, l)$ th element of $P(z)$. Perfect reconstruction for arbitrary $X(z)$ occurs if and only if

$$
\sum_{l=0}^{M-1} W^{-k l} \sum_{s=0}^{M-1} z^{-(l-s)} P_{s, l}\left(z^{M}\right)=\alpha z^{-k_{0}} \delta(k)
$$

for some constant $\alpha$ and some nonnegative integer $k_{0}$. Defining

$$
q(l)=\sum_{s=0}^{M-1} z^{-(l-s)} P_{s, l}\left(z^{M}\right), \quad 0 \leq l \leq M-1,
$$

equation (50) says that the IDFT of the $M$-point sequence $q(l)$ must be an impulse. Accordingly, (50) is equivalent to the requirement

$$
\sum_{s=0}^{M-1} z^{-(l-s)} P_{s, l}\left(z^{M}\right)=\frac{\alpha}{M} z^{-k_{0}}
$$

Let the coefficients of $P_{s, l}(z)$ be denoted $p_{s, l}(n)$, i.e.,

$$
P_{s, l}(z)=\sum_{n=0}^{\infty} p_{s, l}(n) z^{-n}
$$

Equation (52) implies

$$
p_{s, l}(n)=0
$$

for all $n$ except when $n$ satisfies

$$
l-s+n M=k_{0} \text {. }
$$

For every $s$ in $0 \leq s \leq M-1$, there is a unique $l$ in 0 $\leq l \leq M-1$ satisfying (55). This $l$ is given by

$$
l= \begin{cases}s+k_{00}, & s<M-k_{00} \\ s+k_{00}-M, & s \geq M-k_{00}\end{cases}
$$

where $k_{00}$, which satisfies $0 \leq k_{00} \leq M-1$, is defined by

$$
k_{00}=k_{0} \bmod M .
$$

The value of $n$ for which $l$ and $s$ satisfy (56) is given by

$$
n= \begin{cases}k_{01}, & s<M-k_{00} \\ k_{01}+1, & s \geq M-k_{00}\end{cases}
$$

where $k_{01}$ is such that

$$
k_{0}=k_{00}+k_{01} M .
$$

In summary, $\boldsymbol{P}(z)$ takes on the form

$$
\boldsymbol{P}(z)=\frac{\alpha}{M}\left[\begin{array}{ll}
\boldsymbol{O} & z^{-k_{01}} \boldsymbol{I}_{1} \\
z^{-\left(k_{01}+1\right)} \boldsymbol{I}_{2} & \boldsymbol{O}
\end{array}\right]
$$

where $I_{1}$ is the $\left(M-k_{00}\right) \times\left(M-k_{00}\right)$ identity matrix and $I_{2}$ is the $k_{00} \times k_{00}$ identity matrix. If $k_{00}=0$, then $\boldsymbol{P}(z)$ is proportional to the identity matrix, but more generally, a nondiagonal $\boldsymbol{P}(z)$ is permissible. We summarize these results as follows.

Lemma 3.2: Consider a maximally decimated $M$-channel structure as in Fig. 1 with arbitrary $M$. Let $E(z)$ and $\boldsymbol{R}(z)$ be defined in terms of $\left\{H_{k}(z)\right\}$ and $\left\{F_{k}(z)\right\}$, according to (32) and (47), respectively. Then the structure

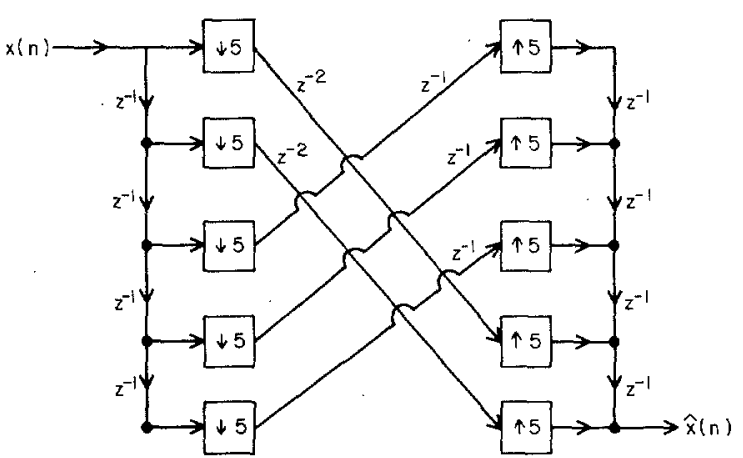

Fig. 11. An example where $\boldsymbol{P}(z)$ is nondiagonal.

gives rise to perfect reconstruction, if and only if the matrix $\boldsymbol{P}(z)$ defined as $\boldsymbol{P}(z)=\boldsymbol{R}(z) \boldsymbol{E}(z)$ is of the form $(60)$. Under such a condition we get

$$
\hat{X}(z)=\frac{\alpha}{M} z^{-\left(k_{0}+M-1\right)} X(z) .
$$

Fig. 11 shows a example where $k_{0}=7$, and $M=5$ so that $k_{00}=2$ and $k_{01}=1$. In this example, $\hat{X}(z)=$ $\alpha z^{-11} X(z) / 5$.

Given an FIR analysis bank (for which $\boldsymbol{E}(z)$ has polynomial entries), there exists an FIR synthesis bank giving rise to perfect reconstruction if $\boldsymbol{R}(z)$ given by

$$
\boldsymbol{R}(z)=\boldsymbol{P}(z) \boldsymbol{E}^{-1}(z)
$$

has polynominal entries. This in turn happens if $\boldsymbol{E}(z)$ has a determinant equal to a power of $z .{ }^{4}$ This is equivalent to saying that det $\boldsymbol{H}(z)$ is a power of $z$. Notice that if $\boldsymbol{E}(z)$ is lossless, then an obvious choice for $\boldsymbol{R}(z)$ in order to obtain perfect reconstruction is

$$
\boldsymbol{R}(z)=z^{-\beta} \boldsymbol{E}^{T}\left(z^{-1}\right)
$$

where $\beta$ is a large enough positive integer such that the RHS of (63) has no positive powers of $z$.

Finally, consider the special case when the analysis filters constitute a uniform DFT bank:

$$
H_{k}(z)=H_{0}\left(z W^{k}\right) \text {. }
$$

Under this condition, with

$$
H_{0}(z)=\sum_{l=0}^{M-1} E_{0 l}\left(z^{M}\right) z^{-l},
$$

we have

$$
H_{k}(z)=\sum_{l=0}^{M-1} E_{0 l}\left(z^{M}\right) W^{-k l} z^{-l}
$$

whence

$$
E_{k l}(z)=W^{-k l} E_{0 l}(z)
$$

${ }^{4}$ Such matrix polynomials are commonly referred to as "unimodular" in the literature [25]. More strictly, a unimodular matrix-polynomial has a constant nonzero determinant. The use of such matrices in perfect-reconstruction banks for arbitrary $M$ has also been independently noted in [15]. 
Thus,

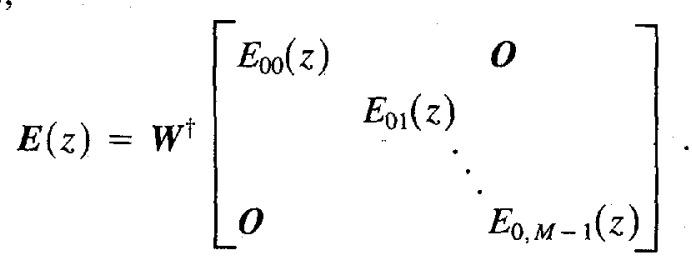

An obvious choice of $\boldsymbol{R}(z)$ in order to get perfect reconstruction is

$$
\boldsymbol{R}(z)=\left[\begin{array}{lll}
E_{00}^{-1}(z) & & \boldsymbol{O} \\
& E_{01}^{-1}(z) & \\
\boldsymbol{O} & & E_{0, M-1}^{-1}(z)
\end{array}\right] \boldsymbol{W}
$$

so that

$$
\boldsymbol{P}(z)=\boldsymbol{R}(z) \boldsymbol{E}(z)=M \cdot \boldsymbol{I}
$$

This choice is indeed practicable, provided the numerators of $E_{0 l}(z)$ have minimum phase. This observation has also been made in some earlier publications [11]-[15]. However, in this special case, the synthesis bank in general is IIR, even with an FIR analysis bank.

\section{Losslessness of AC Matrix in Tree- Structured SB Filter Banks}

The two-channel SB circuit can be used in a tree structure [6] in order to generate perfect-reconstruction QMF banks with $M$ channels when $M$ is a power of two. Fig. 12 is such a demonstration for $M=2^{2}=4$. The quantities $A_{0}(z), A_{1}(z)$ are the analysis filters, and $B_{0}(z), B_{1}(z)$ are the synthesis filters for the basic two-channel prototype, and are related by

$$
\begin{aligned}
& A_{1}(z)=z^{-(N-1)} A_{0}\left(-z^{-1}\right) \\
& B_{0}(z)=z^{-(N-1)} A_{0}\left(z^{-1}\right) \\
& B_{1}(z)=z^{-(N-1)} A_{1}\left(z^{-1}\right)
\end{aligned}
$$

where $N-1$ (which must be odd) is the order of the lowpass filter $A_{0}(z)$. Since the two-channel prototype has perfect-reconstruction property, repeated application of this property shows that the tree structure also has this property. It can be verified that

$$
\hat{X}(z)=z^{-(N-1)(M-1)} X(z)
$$

for such tree structures.

In Section II we saw that the two-channel SB structure has a lossless AC matrix. In Section III we found that for arbitrary $M$, if $\boldsymbol{H}(z)$ is lossless, then there exists a unique way to choose $F_{k}(z)$ so as to obtain perfect reconstruction. In this context, it is natural to raise the following question: does the tree structured SB QMF bank also exhibit a lossless AC matrix? The answer is in the affirmative as one might intuitively expect (but is not entirely obvious) and can be established as follows.

The tree structure can be drawn in an equivalent parallel form as in Fig. 1. Such a redrawing is demonstrated

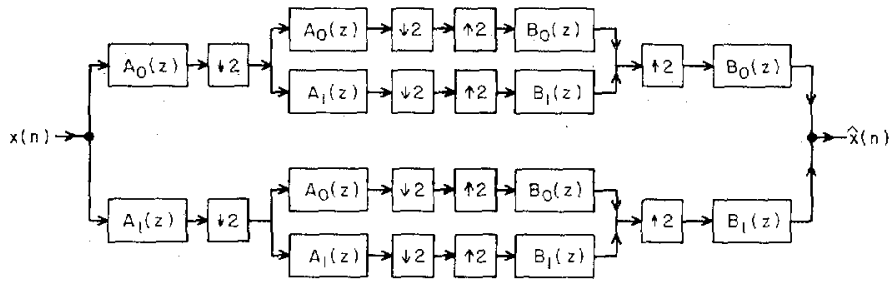

Fig. 12. The tree structure for $M=4$.

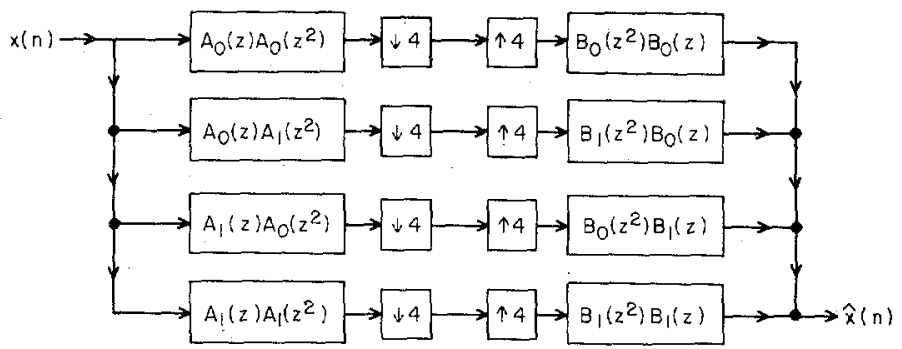

Fig. 13. The parallel equivalent of Fig. 12.

in Fig. 13 for $M=4$, and is justified because of wellknown equivalence relations for multirate systems $[3, \mathrm{ch}$. $3]$. It can be seen, in general, that the $k$ th analysis filter $H_{k}(z)$ of the equivalent parallel structure for $M=2^{L}$ is given by

$$
H_{k}(z)=A_{i 0(k)}(z) A_{i 1(k)}\left(z^{2}\right) \cdots A_{i_{L-1}(k)}\left(z^{M / 2}\right)
$$

where

$$
i_{0}(k) i_{1}(k) \cdots i_{L-1}(k)
$$

is the "binary representation" of the integer $k$, i.e.,

$$
k=\sum_{l=0}^{L-1} i_{l}(k) 2^{L-1-l} .
$$

For example, in Fig. 13, $H_{2}(z)=A_{1}(z) A_{0}\left(z^{2}\right)$. Similarly, the $k$ th synthesis filter $F_{k}(z)$ of the equivalent parallel structure is given by

$$
F_{k}(z)=B_{i 0(k)}(z) B_{i \mid(k)}\left(z^{2}\right) \cdots B_{i_{L-1}(k)}\left(z^{M / 2}\right) .
$$

In yiew of the relations (72) and (73), we obtain from (75) and $(78)$ the following relation:

$$
F_{k}(z)=z^{-\beta} H_{k}\left(z^{-1}\right)
$$

where

$$
\begin{aligned}
\beta & =(N-1)\left(1+2+4+\cdots+2^{L-1}\right) \\
& =(N-1)(M-1) .
\end{aligned}
$$

Thus, the equivalent parallel structure satisfies the relation (45). Moreover, we know that it has perfect-reconstruction property because (74) holds. So, by invoking property 3.4 of Section III, it is immediately clear that the $\mathrm{AC}$ matrix $\boldsymbol{H}(z)$ is indeed lossless.

V. Relation Between $M$ th Band Fir Filters and $M$-Channel Perfect-Reconstruction QMF Banks

Smith and Barnwell [6] and Mintzer [16] have outlined elegant techniques for the design of the transfer function 


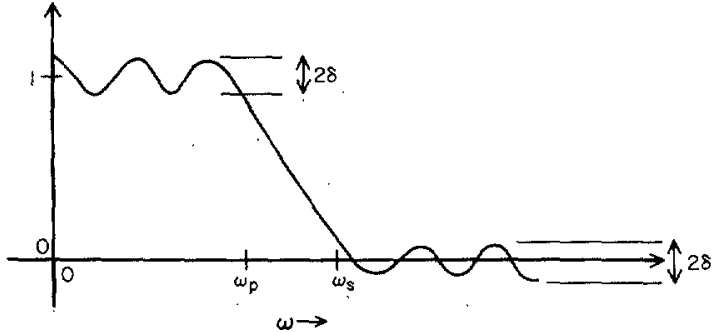

Fig. 14. The amplitude response of a linear-phase half-band filter.

$H_{0}(z)$ in the two-channel SB structure, such that $H_{0}(z)$ has prescribed stopband attenuation, while at the same time satisfying (10) [where $H_{1}(z)$ in $(10)$ is related to $H_{0}(z)$ as in (7)]. Their procedure is based on the design of a linear-phase half-band FIR (low-pass) transfer function $G(z)$ of order $2(N-1)$ [where $N-1$ is the order of $\left.H_{0}(z)\right]$. The frequency response of $G(z)$ has the form

$$
G\left(e^{j \omega}\right)=e^{-j \omega(N-1)} G_{0}\left(e^{j \omega}\right)
$$

where $G_{0}\left(e^{j \omega}\right)$ is the amplitude response and is as shown in Fig. 14. This response exhibits symmetry with respect to $\pi / 2$; in particular, $\omega_{s}=\pi-\omega_{p}$, and $\delta_{1}=\delta_{2}=\delta$. Accordingly, $G(z)$ satisfies

$$
G(z)+(-1)^{N-1} G(-z)=z^{-(N-1)} .
$$

If we now construct $G_{+}(z) \triangleq G(z)+\delta z^{-(N-1)}$, then $G_{+}(z)$ has a positive-valued amplitude response and satisfies

$$
G_{+}(z)+(-1)^{N-1} G_{+}(-z)=(1+2 \delta) z^{-(N-1)} .
$$

Let $H_{0}(z)$ be a spectral factor of $G_{+}(z)$, and define $H_{1}(z)$ as in (7). [Clearly, $H_{1}(z)$ is then a spectral factor of $\left.G_{+}(-z).\right]$ Then (10) is satisfied automatically (up to a scale factor) because of (83). The order of $G(z)$ can be estimated depending upon the required stopband attenuation of $H_{0}(z)$. This then is the essence of the design procedures described in [6] and [16]. The impulse response $g(n)$ of $G(z)$ satisfies the condition $g(n)=0$ if $[n-(N$ - 1)] is a (nonzero) even number. This follows from (82). Accordingly, if $N-1$ is even, then $g(0)=g(2(N$ $-1))=0$ anyway, and hence, $N-1$ can be assumed to be odd without loss of generality.

In designing the analysis bank filters of an $M$-channel perfect-reconstruction QMF structure, the above approach based on half-band filters can be extended. A digital $M$ th band filter [21]

$$
G(z)=\sum_{n=0}^{L-1} g(n) z^{-n}
$$

is a linear-phase (low-pass) FIR filter with cutoff frequency nearly equal to $\pi / M$, and satisfies

$$
g(n)=0, \quad n-\frac{L-1}{2}=\text { nonzero multiple of } M .
$$

We assume $g(n)$ to be real for all $n$. For convenience of discussion, define a zero-phase FIR filter

$$
G_{1}(z)=z^{(L-1) / 2} G(z) .
$$

Because of (84), $G_{1}(z)$ satisfies the condition

$$
\sum_{k=0}^{M-1} G_{1}\left(z W^{-k}\right)=M g\left(\frac{L-1}{2}\right)
$$

where $W=e^{-j 2 \pi / M}$. Assume that $G_{1}\left(e^{j \omega}\right)$ (which is real) is also nonnegative for all $\omega$ (if this is not true, it can be ensured simply by adding $\epsilon z^{-(L-1) / 2}$ to $G(z)$ where $\epsilon$ is sufficiently large). We can now define (causal) spectral factors $H_{k}(z)$ of $G_{1}\left(z W^{-k}\right)$, for each $k$ :

$$
\begin{aligned}
H_{k}(z) H_{k, *}\left(z^{-1}\right)=G_{1}\left(z W^{-k}\right), & \\
& 0 \leq k \leq M-1 .
\end{aligned}
$$

In view of (86), the spectral factors therefore satisfy the condition

$$
\sum_{k=0}^{M-1} H_{k, *}\left(z^{-1}\right) H_{k}(z)=M g\left(\frac{L-1}{2}\right) .
$$

This condition is an extension of (10) which was satisfied by the two-channel SB structure. Accordingly, if we neglect the aliasing effects in an $M$-channel QMF bank, and design $H_{k}(z)$ in the above manner, then the choice of synthesis filters as in (45) ensures perfect reconstruction. Perfect-reconstruction results, under the assumption that aliasing is negligible, can be found in [35].

Let us now explore the aliasing problem when the filters are chosen in this particular manner. Replacing $z$ with $z W^{-l},(88)$ implies

$$
\sum_{l=0}^{M-1} H_{k, *}\left(z^{-1} W^{l}\right) H_{k}\left(z W^{-l}\right)=M g\left(\frac{L-1}{2}\right) .
$$

Consider an $M$-channel QMF bank as in Fig. 1 with $H_{k}(z)$ defined in the above manner. If the corresponding AC matrix $\boldsymbol{H}(z)(34)$ can be forced to be lossless, then we can immediately obtain the synthesis bank that would enable perfect reconstruction. Recall that losslessness of $\boldsymbol{H}(z)$ is equivalent to

$$
\boldsymbol{H}_{*}^{T}\left(z^{-1}\right) \boldsymbol{H}(z)=\boldsymbol{I},
$$

i.e.,

$$
\sum_{s=0}^{M-1} H_{k, *}\left(z^{-1} W^{s}\right) H_{l}\left(z W^{-s}\right)=\delta(k-l) .
$$

Since $H_{k}(z)$ have been constructed by spectral factorization of an $M$ th band filter, they satisfy (89). Hence, (91) is automatically satisfied for $k=l$ (except for a scale factor of no consequence). Now, if (91) holds for all $k, l$ with $0 \leq k, l \leq M-1$, perfect reconstruction is possible. Let us next examine the side effects of satisfying (91) for $k \neq$ $l$. For example, let $k=1, l=0$, then (91) implies

$$
\sum_{s=0}^{M-1} H_{1, *}\left(z^{-1} W^{s}\right) H_{0}\left(z W^{-s}\right)=0 .
$$


(a)

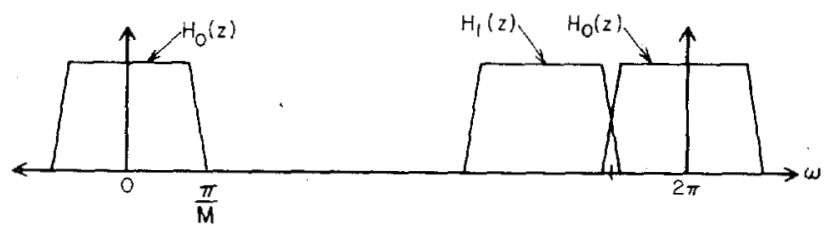

(b)

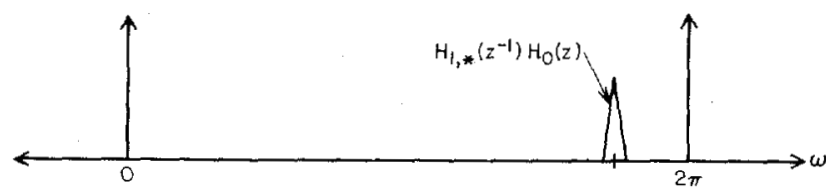

(c)

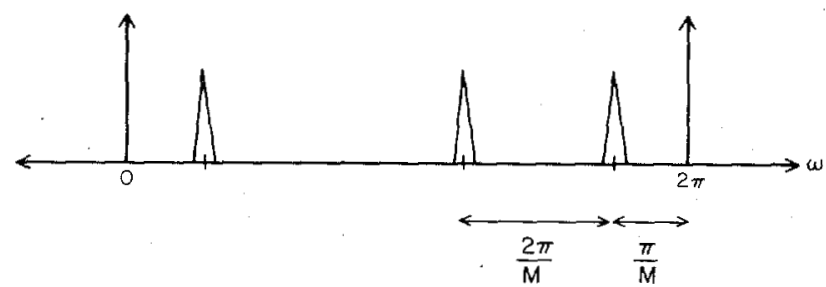

Fig. 15. Pertaining to design based on $M$ th-band filters.

The magnitudes of $H_{0}(z), H_{1}(z)$, and $H_{1, *}\left(z^{-1}\right) H_{0}(z)$ on the unit circle are sketched in Fig. 15. In Fig. 15(c) is shown the typical appearance of the magnitudes of the quantities appearing in the summation of (92). It is clear that if $H_{0}(z)$ is a good low-pass filter with cutoff $\pi / M$, then there is hardly an overlap between the successive waveforms in Fig. 15(c), unless $M=2$. Accordingly, for $M>2$, it is not possible to satisfy $(92)$, unless $H_{0}(z)$ is a "poor" low-pass function which would permit overlap between adjacent spectral shapes in Fig. 15(c). But for subband coding applications, the stopband rejection of $H_{k}(z)$ is of crucial importance [3].

In conclusion, if we design $H_{k}(z)$ by spectral factorizing an $M$ th band filter, and if $H_{0}(z)$ is a sharp-cutoff filter with reasonably large stopband attenuation, then $H(z)$ cannot be made lossless unless $M=2$. Now, property 3.4 says that if (45) holds, then losslessness of $\boldsymbol{H}(z)$ is a necessary condition for perfect reconstruction. Accordingly, if (45) holds, and if $H_{k}(z)$ are "good" filters obtained as above, then since $\boldsymbol{H}(z)$ cannot be lossless, we cannot have perfect reconstruction either. In conclusion, if $H_{k}(z)$ are designed based on $M$ th band filters, and if $F_{k}(z)$ are chosen as in (45), we cannot have perfect reconstruction unless $H_{k}(z)$ are poor filters (or unless we choose to ignore aliasing).

Finally, notice that the spectral factors $H_{k}(z)$ defined in (87) will have complex coefficients except when $k=$ 0 , and hence, the subband signals for such a scheme would be complex, even for real signals. This would not always be desirable.

In the next section we turn our attention to better design procedures which ensure that $\boldsymbol{H}(z)$ is lossless (hence permitting perfect reconstruction), at the same time permitting $H_{0}(z)$ to have arbitrarily sharp cutoff and large stopband attenuation. In addition, all transfer functions $H_{k}(z)$ have real coefficients.

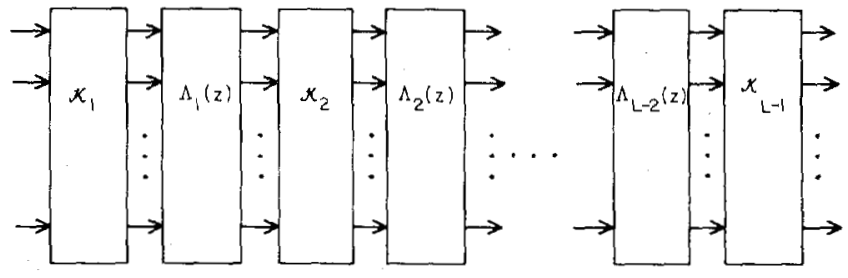

Fig. 16. A simple means of obtaining an $M \times M$ lossless matrix $\boldsymbol{E}(z)$.

\section{Design Based on Numerical Optimization of Lossless FIR Lattice Structures}

A technique has recently been proposed [18]-[20] for the implementation of FIR filters and filter banks based on lossless building blocks. The basic ideas of such a technique can be exploited in order to design the analysis (and synthesis) bank filters of the perfect-reconstruction QMF structure. We include here a self-contained but brief description of the procedure for doing this.

Consider again the QMF structure of Fig. 7. According to the results of Section III, if the $M$-input $M$-output system $\boldsymbol{E}(z)$ is FIR and lossless, ${ }^{5}$ then Fig. 7 represents a perfect-reconstruction QMF bank. From the definition of losslessness, it is clear that a cascade of lossless systems is lossless. For example, if $\boldsymbol{E}(z)=\boldsymbol{E}_{1}(z) \boldsymbol{E}_{2}(z)$, and if $E_{1}(z)$ and $E_{2}(z)$ are lossless, then

$$
\tilde{E} \boldsymbol{E}(z)=\tilde{E}_{2}(z) \tilde{E}_{1}(z) \boldsymbol{E}_{1}(z) \boldsymbol{E}_{2}(z)=\tilde{\boldsymbol{E}}_{2}(z) \boldsymbol{E}_{2}(z)=\boldsymbol{I}
$$

A simple means, therefore, of obtaining an $M \times M$ lossless system $\boldsymbol{E}(z)$ is indicated in Fig. 16, which is a cascade of two kinds of lossless building blocks. The building blocks $\mathrm{K}_{i}$ are constant unitary matrices, i.e.,

$$
\mathrm{K}_{i}{ }^{\dagger} \mathrm{K}_{i}=\boldsymbol{I}, \quad 1 \leq i \leq L-1 .
$$

In this section we consider QMF banks with real coefficients, hence, $K_{i}$ have real coefficients (i.e., they are orthogonal matrices).

The second type of building blocks $\Lambda_{i}(z)$ which separate successive $K_{i}$ are diagonal matrices with delay elements. The diagonal nature ensures that $\Lambda_{i}(z)$ are lossless. For example, a typical $\Lambda_{i}(z)$ for $M=3$ could be

$$
\Lambda_{i}(z)=\left[\begin{array}{lll}
1 & 0 & 0 \\
0 & 1 & 0 \\
0 & 0 & z^{-1}
\end{array}\right] \text {. }
$$

This choice of $\Lambda_{i}(z)$ is clearly not the only possible diagonal matrix of delay elements, and is meant to serve as an example. Our numerical example will be based on (94) because the form (94) has been used in an earlier paper [20] under a different context. The "best' choice of the diagonal matrix $\Lambda_{i}(z)$ that maximizes attenuation provided by $H_{k}(z)$ is not clear at this point in time.

\footnotetext{
"In the case of FIR filters, the term "lossless" is synonymous with "unitary on the unit circle of $z$-plane" because stability is automatically guar anteed.
} 
If we employ the $M$-input $M$-output system of Fig. 16 in place of $\boldsymbol{E}(z)$ in Fig. 7, then perfect reconstruction is guaranteed! It only remains to adjust the parameters of the matrices $\mathrm{K}_{i}$ such that $H_{k}(z)$ will have good attenuation characteristics. This can be accomplished by nonlinear optimization techniques, and some details are included in this section. It should be noticed that once $H_{k}(z)$ are computed in this fashion, the designer has the choice of either using the structure of Fig. 7 (with $\boldsymbol{E}(z)$ as in Fig. $16)$, or simply implementing $H_{k}(z)$ in direct form and obtaining the structure of Fig. 1 [the filters $F_{k}(z)$ can be obtained from $H_{k}(z)$ as in (45)]. In either case, the reconstruction is perfect (as long as signal rounding effects and coefficient quantization are small enough to be ignored).

\section{A. An Optimization Procedure}

A simple way to generate an $M \times M$ orthogonal matrix $\mathrm{K}_{i}$ is as a sequence of planar rotations. For example,

$$
\begin{aligned}
\mathrm{K}_{i}= & {\left[\begin{array}{ccc}
\cos \theta_{1, i} & \sin \theta_{1, i} & \boldsymbol{O} \\
\sin \theta_{1, i} & -\cos \theta_{1, i} & \boldsymbol{O} \\
\boldsymbol{O} & \boldsymbol{O} & \boldsymbol{I}_{1}
\end{array}\right] } \\
& {\left[\begin{array}{llll}
1 & 0 & 0 & \boldsymbol{O} \\
0 & \cos \theta_{2, i} & \sin \theta_{2, i} & \boldsymbol{O} \\
0 & \sin \theta_{2, i} & -\cos \theta_{2, i} & \boldsymbol{O} \\
\boldsymbol{O} & \boldsymbol{O} & \boldsymbol{O} & \boldsymbol{I}_{2}
\end{array}\right] } \\
& \cdots\left[\begin{array}{ccc}
\boldsymbol{I}_{M-1} & \boldsymbol{O} & \boldsymbol{O} \\
\boldsymbol{O} & \cos \theta_{M-1, i} & \sin \theta_{M-1, i} \\
\boldsymbol{O} & \sin \theta_{M-1, i} & -\cos \theta_{M-1, i}
\end{array}\right]
\end{aligned}
$$

where $\boldsymbol{I}_{k}$ are identity matrices of appropriate dimensions (not necessarily equal to the subscript $k$ ). It should be realized that (95) does not represent a most general $M \times$ $M$ orthogonal matrix (which would involve more than $M$ - 1 rotational angles [26]), and is merely intended as a possibility. Assuming that each $\boldsymbol{K}_{i}$ in Fig. 16 has the above form, an objective function $\phi$ can be formulated which measures the filtering accuracy of $H_{k}(z)$ (such as stopband attenuation). Such a function depends upon $\theta_{k, i}, 1$ $\leq k \leq M-1,1 \leq i \leq L-1$ in a nonlinear fashion, and can be optimized by employing standard gradient algorithms.

We now demonstrate this procedure with an example for the case of three channels.

A Design Example: Let $M=3$. In this example, we constrain $E(z)$ to be as in Fig. 17, so that $\Lambda_{i}(z)$ are as in (94). We restrict $K_{i}$ to be as in (95), i.e.,

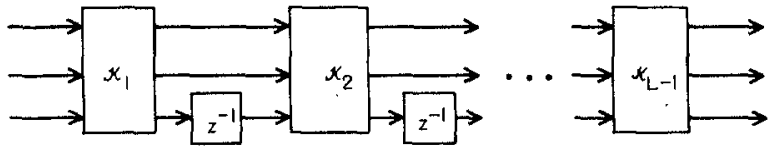

Fig. 17. The $3 \times 3$ lossless $E(z)$ for the design example.

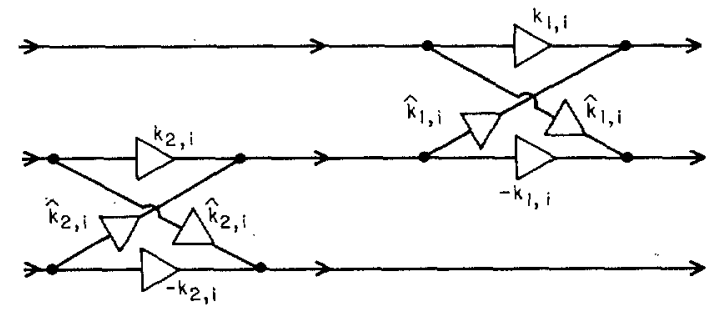

Fig. 18. The lattice structure implementing $\mathrm{K}_{i}$.

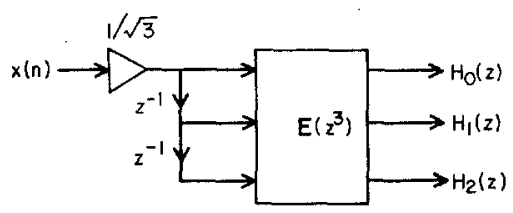

Fig. 19. The analysis bank for the 3-channel case.

$$
\begin{aligned}
K_{i}= & {\left[\begin{array}{ccc}
\cos \theta_{1, i} & \sin \theta_{1, i} & 0 \\
\sin \theta_{1, i} & -\cos \theta_{1, i} & 0 \\
0 & 0 & 1
\end{array}\right] } \\
& {\left[\begin{array}{lll}
1 & 0 & 0 \\
0 & \cos \theta_{2, i} & \sin \theta_{2, i} \\
0 & \sin \theta_{2, i} & -\cos \theta_{2, i}
\end{array}\right] . }
\end{aligned}
$$

The lattice structure implementing $\mathrm{K}_{i}$ is shown in Fig. 18, where $k_{1, i} \doteq \cos \theta_{1, i}, \hat{k}_{1, i}=\sin \theta_{1, i}, k_{2, i}=\cos \theta_{2, i}, \hat{k}_{2, i}$ $=\sin \theta_{2, i}$. The three transfer functions $H_{0}(z), H_{1}(z)$, and $H_{2}(z)$ of the analysis bank (Fig. 19) are automatically guaranteed to satisfy the condition

$$
\left|H_{0}\left(e^{j \omega}\right)\right|^{2}+\left|H_{1}\left(e^{j \omega}\right)\right|^{2}+\left|H_{2}\left(e^{j \omega}\right)\right|^{2}=1
$$

because the losslessness of $\boldsymbol{E}(z)$ [induced by the orthogonality of (96)] ensures that the AC matrix $\boldsymbol{H}(z)$ is lossless. We wish the frequency responses to be of the form in Fig. 20 and accordingly formulate an objective function

$$
\begin{aligned}
\phi= & \int_{(\pi / 3)+\epsilon}^{\pi}\left|H_{0}\left(e^{j \omega}\right)\right|^{2} d \omega+\int_{0}^{(2 \pi / 3)-\epsilon}\left|H_{2}\left(e^{j \omega}\right)\right|^{2} d \omega \\
& +\int_{0}^{(\pi / 3)-\epsilon}\left|H_{1}\left(e^{j \omega}\right)\right|^{2} d \omega \\
& +\int_{(2 \pi / 3)+\epsilon}^{\pi}\left|H_{1}\left(e^{j \omega}\right)\right|^{2} d \omega .
\end{aligned}
$$




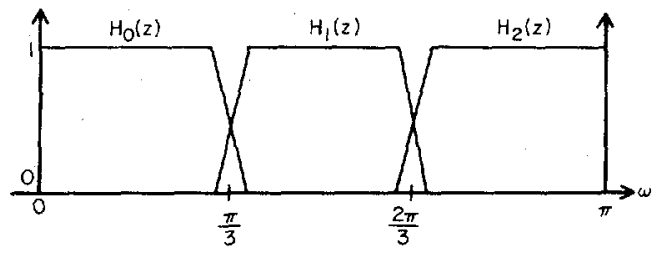

Fig. 20. The magnitude responses desired in the analysis bank.

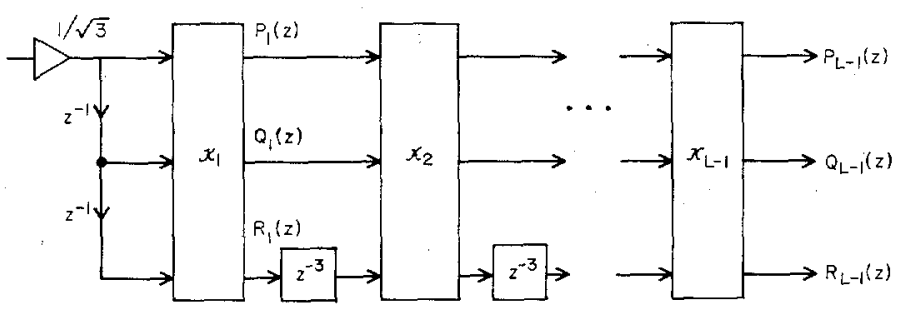

Fig. 21. The structure for the optimization of analysis filters.

The quantity $\epsilon$ depends on the desired stopband edges. The function $\phi$ involves only the stopband energies of the various transfer functions. However, if we minimize $\phi$, it automatically ensures good passband responses, since the constraint (97) is enforced by the structure of Fig. 17. For example, in the frequency region $(0, \pi / 3-\epsilon)$, $\left|H_{1}\left(e^{j \omega}\right)\right|^{2}$ and $\left|H_{2}\left(e^{j \omega}\right)\right|^{2}$ are "small," since $\phi$ is minimized. Accordingly, $\left|H_{0}\left(e^{j \omega}\right)\right|$ is close to unity, because of (97).

The minimization of $\phi$ can be accomplished by invoking a nonlinear optimization software package. We employed a routine (called ZXMWD) of the IMSL software package [27], which is based on a modified quasi-Newton technique [28]. In order to invoke this routine, the user supplies a subroutine that computes $\phi$ for a given set of values of $\theta_{k, i}$. (The user is not required to supply the gradient.) Such a subroutine should first compute the coeffi-. cients of the polynomials $H_{0}(z), H_{1}(z), H_{2}(z)$, and then evaluate $\phi$. The computation of the polynomial coefficients can be done recursively as follows (see Fig. 21).

Recursion:

$$
\begin{aligned}
P_{m}(z)= & k_{1, m} P_{m-1}(z)+k_{2, m} \hat{k}_{1, m} Q_{m-1}(z) \\
& +z^{-3} \hat{k}_{2, m} \hat{k}_{1, m} R_{m-1}(z) \\
Q_{m}(z)= & \hat{k}_{1, m} P_{m-1}(z)-k_{2, m} k_{1, m} Q_{m-1}(z) \\
& -z^{-3} \hat{k}_{2, m} k_{1, m} R_{m-1}(z) \\
R_{m}(z)= & \hat{k}_{2, m} Q_{m-1}(z)-z^{-3} k_{2, m} R_{m-1}(z) .
\end{aligned}
$$

Initialization:

$$
\begin{aligned}
& P_{1}(z)=\left(k_{1,1}+z^{-1} k_{2,1} \hat{k}_{1,1}+z^{-2} \hat{k}_{2,1} \hat{k}_{1,1}\right) / \sqrt{3} \\
& Q_{1}(z)=\left(\hat{k}_{1,1}-z^{-1} k_{2,1} k_{1,1}-z^{-2} \hat{k}_{2,1} k_{1,1}\right) / \sqrt{3} \\
& R_{1}(z)=\left(z^{-1} \hat{k}_{2,1}-z^{-2} k_{2,1}\right) / \sqrt{3} .
\end{aligned}
$$

In the above equations, $k_{i, m}=\cos \theta_{i, m}$ and $\hat{k}_{i, m}=\sin \theta_{i, m}$. At the end of the recursion we obtain the polynomials

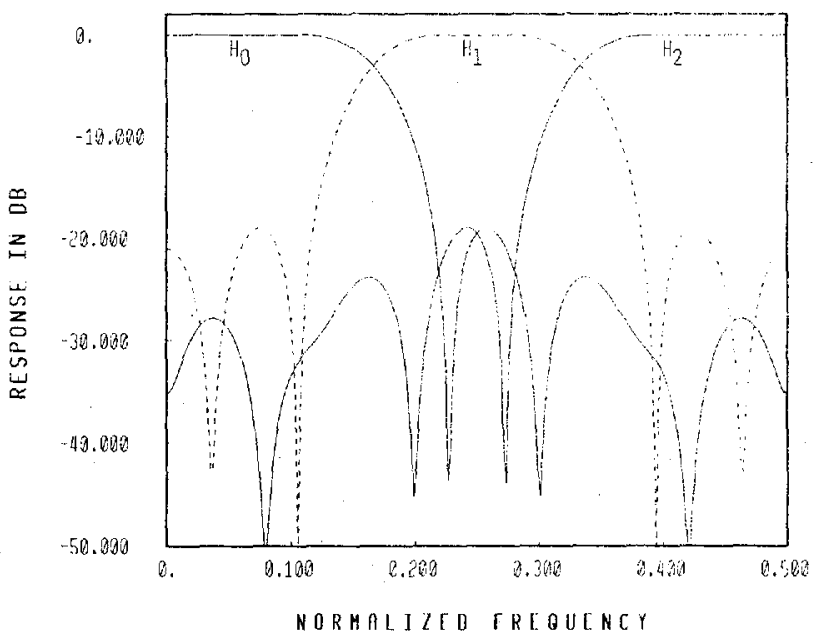

Fig. 22. Magnitude response plots for the optimized analysis filters.

$H_{0}(z)=P_{L-1}(z), H_{1}(z)=Q_{L-1}(z), \quad H_{2}(z)=R_{L-1}(z)$

which enables us to compute $\phi$ in (98). The order of $H_{k}(z)$ is $N-1=3(L-2)+2$. Fig. 22 shows the magnitude response plots of the three optimized analysis filters $H_{k}(z)$, for an example where $L-1=5$. The number of theta parameters is 10 , and the orders of $H_{k}(z)$ are $N-$ $1=3 \times(L-2)+2=14$. Table I shows the parameters $k_{i, m}$ and $\hat{k}_{i, m}$, whereas Table II shows the impulse response coefficients of $H_{k}(z)$. The synthesis filters can be taken to be $F_{k}(z)=z^{-14} H_{k}\left(z^{-1}\right)$, in order to obtain perfect reconstruction. (Note that $F_{k}(z)$ have the same order as the analysis filters.) Even though the attenuation in Fig. 22 is not sufficient for standard QMF applications, this can be improved by increasing the number of stages $L-$ 1 in Fig. 17 so that the analysis bank has a higher order.

The way in which the objective function is defined in (98) is such that, upon convergence of the optimization algorithm, $\left|H_{2}\left(e^{j \omega}\right)\right|$ is the image of $\left|H_{0}\left(e^{j \omega}\right)\right|$ with respect to $\pi / 2$, and moreover, $\left|H_{1}\left(e^{j \omega}\right)\right|$ is symmetric with respect to $\pi / 2$. This can also be seen from Fig. 22. Accordingly, once the optimization converges, we will have $\left|H_{2}\left(e^{j \omega}\right)\right|=\left|H_{0}\left(-e^{j \omega}\right)\right|$. This does not necessarily imply $H_{2}(z)= \pm H_{0}(-z)$, and the fact that this is (approximately) the case in Table II is only a coincidence. In fact, the lattice structure itself does not even impose the restriction $\left|H_{2}\left(e^{j \omega}\right)\right|=\left|H_{0}\left(-e^{j \omega}\right)\right|$. Next, from Table II we see that $\left|H_{2}\left(e^{j \omega}\right)\right|$ is only approximately equal to $\left|H_{0}\left(-e^{j \omega}\right)\right|$, but this relation can be made more exact simply by requesting a more stringent convergence criterion for optimization. We wish to emphasize, however, that perfect-reconstruction property will be exactly satisfied even if the optimization is inaccurate, simply by virtue of the fact that the transfer functions are derived from the lattice structure. Thus, perfect reconstruction is structurally induced. The values of $\theta_{i, j}$ determine only the shapes of the individual $H_{k}(z)$ 's. 
TABLE I

The Value of the Lattice Coefficients in the Optimized ANalysis

Bank. Number of Sections $L-1=5$; Filter Order $N-1=14$

\begin{tabular}{|c|c|c|c|c|}
\hline $\mathrm{m}$ & $k_{1}, m$ & $\hat{k}_{1, m}$ & $k_{2, m}$ & $\widehat{k}_{2, m}$ \\
\hline $\begin{array}{l}1 \\
2 \\
3 \\
4 \\
5\end{array}$ & $\begin{array}{r}-0.70922170 \mathrm{~d}+\infty \\
0.95631350 \mathrm{~d}+\infty \\
0.15709290 \mathrm{~d}+\infty \\
0.32816120 \mathrm{~d}+\infty \\
-0.1137000 \mathrm{~d}-03\end{array}$ & $\begin{array}{r}0.70498552 d+\infty \\
0.29234310 d+\infty \\
0.98758383 d+\infty \\
-0.94462174 d+\infty \\
0.99999999 d+\infty\end{array}$ & $\begin{array}{l}-0.93600000 d-\infty 4 \\
-0.35865230 d+\infty \\
-0.99999990 d+\infty \\
-0.15896640 d+\infty \\
-0.70708230 d+\infty\end{array}$ & $\begin{array}{r}-0.10000000 \mathrm{~d}+01 \\
-0.93347122 \mathrm{~d}+\infty \\
0.44721358 \mathrm{~d}-03 \\
-0.98728399 \mathrm{~d}+\infty \\
0.70713126 \mathrm{~d}+\infty\end{array}$ \\
\hline
\end{tabular}

TABLE II

IMPULSE RESPONSE COEFFICIENTS OF THE OPTIMIZED ANALYSIS BANK FILTERS

\begin{tabular}{|c|c|c|c|}
\hline$n$ & $n_{0}(n)$ & $h_{1}(n)$ & $h_{2}(n)$ \\
\hline $\begin{array}{r}0 \\
1 \\
2 \\
3 \\
4 \\
5 \\
6 \\
7 \\
8 \\
9 \\
10 \\
11 \\
12 \\
13 \\
14\end{array}$ & $\begin{array}{r}-0.42975335986199 \mathrm{~d}-01 \\
0.13938012584211 \mathrm{~d}-04 \\
0.14891039020466 \mathrm{~d}+\infty \\
0.29711060779911 \mathrm{~d}+\infty \\
0.35375400443686 \mathrm{~d}+\infty 0 \\
0.26733022920753 \mathrm{~d}+\infty 0 \\
0.87062827411073 \mathrm{~d} \times 01 \\
-0.52122033341321 \mathrm{~d}-01 \\
-0.87593136078151 \mathrm{~d}-01 \\
-0.42709632397534 \mathrm{~d} \times 01 \\
0.47426369238237 \mathrm{~d}-01 \\
0.42961831070467 \mathrm{~d}-01 \\
0 . \quad \text { d+OO } \\
-0.23276534497968 \mathrm{~d}-01 \\
0.21786836385535 \mathrm{~d}-05\end{array}$ & $\begin{array}{r}-0.92770412381267 d-01 \\
0.82043979687156 d-06 \\
0.87653824068124 d-02 \\
-0.89793582734887 d-05 \\
0.18640257224072 d+\infty \\
-0.43884653241662 d-04 \\
-0.35433035625168 d+\infty \\
-0.21534684870289 d-03 \\
0.35645950964714 d+\infty \\
-0.48560852349886 d-05 \\
-0.19310826169468 d+\infty 0 \\
0.22960198324688 d-04 \\
0.0 \quad d+\infty \\
-0.26465419895258 d-05 \\
0.24771633130473 d-09\end{array}$ & $\begin{array}{r}0.42988860238211 d-01 \\
-0.13939070914603 d-04 \\
-0.14892169715324 d+\infty \\
0.29732024389502 d+\infty \\
-0.35374966894441 d+\infty \\
0.26709728401356 d+\infty \\
-0.87063835222800 d-01 \\
-0.52084419980668 d-01 \\
0.87579833045549 d-01 \\
-0.42706675473313 d-01 \\
-0.47471783144698 d-01 \\
0.42967738845495 d-01 \\
0 . \\
-0.23274922989163 d-01 \\
0.21785328013287 d-05\end{array}$ \\
\hline
\end{tabular}

TABLE III

IMPULSE RESPONSE OF THE COMPOSITE SYSTEM

\begin{tabular}{|c|c|c|c|}
\hline & & he sequence $\hat{x}(n)$ & \\
\hline$n$ & for $x(n)=\delta(n)$ & for $x(n)=\delta(n-1)$ & for $x(n)=5(n-2)$ \\
\hline 0 & 0.0000000000 & 0. & 0 \\
\hline 1 & -0.000000000 & 0.000000000 & \\
\hline 2 & -0.000000000 & -0.000000000 & 0.000000000 \\
\hline 3 & 0.000000000 & -0.0000000000 & -0.000000000 \\
\hline 4 & -0.000000000 & 0.000000000 & -0.000000000 \\
\hline 5 & -0.000000000 & -0.0000000000 & 0.000000000 \\
\hline 6 & 0.000000000 & -0.000000000 & -0.000000000 \\
\hline 7 & -0.000000000 & 0.000000000 & -0.000000000 \\
\hline 8 & 0.000000000 & .0 .000000000 & 0.000000000 \\
\hline 9 & 0.000000000 & 0.0000000000 & -0.000000000 \\
\hline 10 & -0.000000000 & 0.000000000 & 0.000000000 \\
\hline 11 & -0.000000000 & -0.000000000 & 0.000000000 \\
\hline 12 & 0.000000000 & -0.000000000 & -0.000000000 \\
\hline 13 & -0.000000000 & 0.000000000 & .0 .000000000 \\
\hline 14 & 1.000000000 & -0.000000000 & 0.000000000 \\
\hline 15 & 0.000000000 & 1.000000000 & -0.0000000000 \\
\hline 16 & 0.000000000 & 0.000000000 & 1.000000000 \\
\hline 17 & 0.000000000 & 0.000000000 & -0.000000000 \\
\hline 18 & -0.000000000 & 0.000000000 & 0.000000000 \\
\hline 19 & 0.000000000 & -0.000000000 & 0.000000000 \\
\hline 20 & -0.000000000 & 0,000000000 & 0.0000000000 \\
\hline 21 & -0.000000000 & - 0000000000 & 0.000000000 \\
\hline 22 & 0.000000000 & -0.000000000 & -0.000000000 \\
\hline 23 & -0.000000000 & 0.000000000 & -0.0000000000 \\
\hline 24 & -0.000000000 & -0.000000000 & 0.0000000000 \\
\hline 25 & 0.000000000 & .0 .000000000 & -0.000000000 \\
\hline 26 & 0.000000000 & 0.000000000 & -0.000000000 \\
\hline 27 & -0.000000000 & 0.000000000 & 0.000000000 \\
\hline 28 & 0.000000000 & -0.000000000 & 0.000000000 \\
\hline 29 & 0 & 0.000000000 & -0.000000000 \\
\hline 30 & 0. & 0. & 0.000000000 \\
\hline
\end{tabular}

In order to demonstrate the perfect-reconstruction property of the QMF bank characterized by the parameters in Tables I and II, the complete system of Fig. 7 has been
TABLE IV

AN ARbitrary Input SEQuence $x(n)$ AND the ReConstructed Sequence $\hat{x}(n)$ FOR THE DESIGN EXAMPLE. Here $\hat{x}(n+N-1)$ IS SHOWN, IN ORDER TO ALIGN THE SAMPLES

\begin{tabular}{crr}
$n$ & \multicolumn{1}{c}{$x(n)$} & \multicolumn{1}{c}{$\hat{x}(n+N-1)$} \\
\hline 1 & 1.000000000 & 1.000000000 \\
2 & 0.500000000 & 0.500000000 \\
3 & 0.600000000 & 0.600000000 \\
4 & 0.300000000 & 0.300000000 \\
5 & -0.300000000 & -0.300000000 \\
6 & 0.400000000 & 0.400000000 \\
7 & 0.350000000 & 0.350000000 \\
8 & 3.12000000 & 3.120000000 \\
9 & 1.003000000 & 1.003000000 \\
10 & -0.450000000 & -0.450000000 \\
\hline
\end{tabular}

simulated on a computer. With the input $x(n)$ taken as an impulse function $\delta(n-k)$ with $k=0,1$, and 2 , respectively, the response $\hat{x}(n)$ is shown in the three columns of Table III. This response is clearly an impulse (up to an accuracy of at least 10 significant decimal digits). Since the columns of Table III are successively shifted versions, the time invariance of the complete system is demonstrated (thereby demonstrating alias cancellation). Table IV shows an arbitrary input $x(n)$ and the reconstructed signal $\hat{x}(n)$. It is clear that $\hat{x}(n)$ is a delayed version of $x(n)$. These two tables thus demonstrate the perfect-reconstruction property. Finally, Fig. 23 shows a plot of $\left|T\left(e^{j \omega}\right)\right|=\left|H_{0}\left(e^{j \omega}\right)\right|^{2}+\left|H_{1}\left(e^{j \omega}\right)\right|^{2}+\left|H_{2}\left(e^{j \omega}\right)\right|^{2}$, where $T(z)$ is the composite transfer function 


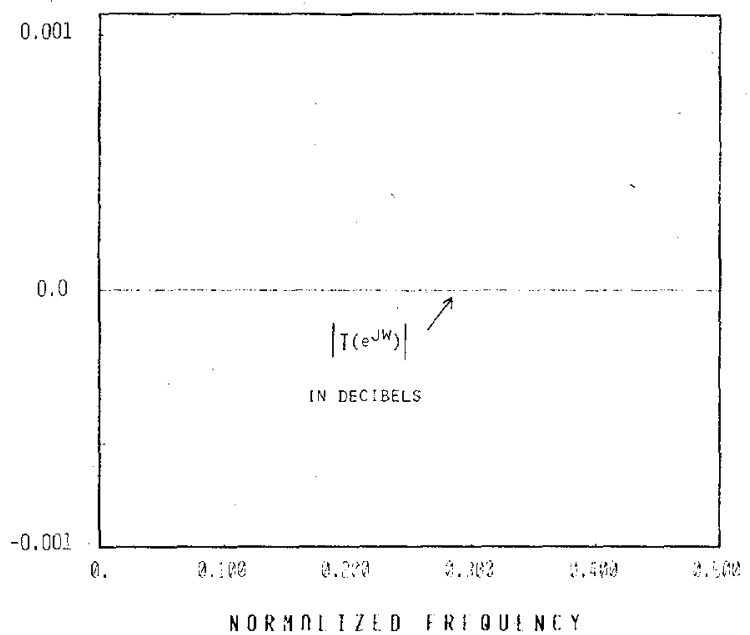

Fig. 23. The composite response in decibels for the design example.

$\hat{X}(z) / X(z)$. Evidently, $\left|T\left(e^{j \omega}\right)\right|=1$ for all $\omega$, in accordance with perfect-reconstruction property.

\section{B. Comments and Further Generalizations}

In a practical implementation of a perfect-reconstruction system, there are three additional sources of error. First, the elements characterizing the matrices $\mathrm{K}_{i}$ have to be quantized, which means they will not be exactly orthogonal. Second, there is computational roundoff noise when the analysis and synthesis banks are digitally implemented. Finally, since the subband signals themselves are encoded before transmission (and subsequently decoded at the synthesis end), the coding error gets reflected in the reconstructed signal. These three errors are ignored in this sequel, as they require further study.

A considerable amount of work remains to be done in connection with improved optimal designs of $H_{k}(z)$. First, the matrices in (95) and (96) are not the most general orthogonal forms. (For example, a general $3 \times 3$ orthogonal matrix requires three planar angles in order to be completely characterized.) More general orthogonal forms can offer improved stopband attenuations for the same order $N-1$. Second, there is no reason in practice to restrict oneself to the simplified form of $\Lambda_{i}(z)$ as in (94). Finally, losslessness of $\boldsymbol{H}(z)$ (which is the basis of obtaining the above optimization procedure) is itself only a sufficient (rather than necessary) condition for perfection of reconstruction, and the question is whether we can obtain more efficient designs in other ways.

As in most nonlinear optimization problems, it is possible to have local extrema, and it is judicious to try out several initial parameter estimates in order to obtain a solution that is (reasonably close to) the global minimum.

Explicit computation of the gradient of $\phi$ (rather than gradient-approximation using differences) is often preferred [28] in the procedure for optimization of $\phi$. Gradient computation is rendered easy in the case of our objective function because of the way in which the $\theta_{k, i}$ parameters enter (95) and (96). For example, $\partial \phi / \partial \theta_{1, i}$ can be found simply by replacing the matrix

$$
\left[\begin{array}{ccc}
\cos \theta_{1, i} & \sin \theta_{1, i} & 0 \\
\sin \theta_{1, i} & -\cos \theta_{1, i} & 0 \\
0 & 0 & 1
\end{array}\right]
$$

with the derivative

$$
\left[\begin{array}{lll}
-\sin \theta_{1, i} & \cos \theta_{1, i} & 0 \\
\cos \theta_{1, i} & \sin \theta_{1, i} & 0 \\
0 & 0 & 0
\end{array}\right]
$$

The objective function obtained by replacing (102) with (103) is precisely the gradient $\partial \phi / \partial \theta_{1, i}$. In the design example reported above, this gradient computation has not been utilized.

Summarizing, the design results presented in this subsection are by no means the best in the sense that more general orthogonal matrices can be used during optimization, and $\Lambda_{i}(z)$ can be more general than in (94). The example is meant only to demonstrate the basic result on perfect reconstruction in maximally decimated QMF banks for arbitrary $M$. The scope for improvement is wide.

\section{Signal Reconstruction in QMF Banks with ReCURSIVE FiLTeRs}

In Section III it was indicated that, under certain (rather stringent) restrictions, it is possible to obtain perfect-reconstruction IIR QMF banks. More useful solutions in the IIR case can be obtained if phase distortion in the reconstruction process can be tolerated. This can be accomplished by forcing $T(z)$ in (2) to be (stable) and all-pass. Examples can be found in [11]-[13], [32], and [34]. In this section we wish to indicate a general means of accomplishing this, based on the losslessness concept.

The inverse of the $\mathrm{AC}$ matrix is

$$
\boldsymbol{H}^{-1}(z)=\frac{\operatorname{Adj} \boldsymbol{H}(z)}{A(z)}
$$

where $A(z)=\operatorname{det} \boldsymbol{H}(z)$. Clearly, $A(z)$ is stable (assuming $H_{k}(z)$ are stable) and the entries in $\operatorname{Adj} \boldsymbol{H}(z)$ are stable, but $\boldsymbol{H}^{-1}(z)$ is not necessarily stable unless the zeros of $A(z)$ are restricted to be strictly inside the unit circle. Let us now consider the consequence of choosing the synthesis filters $F_{k}(z)$ to be

$$
f(z) \triangleq\left[\begin{array}{c}
F_{0}(z) \\
F_{1}(z) \\
\vdots \\
F_{M-1}(z)
\end{array}\right]=\operatorname{Adj} \boldsymbol{H}(z)\left[\begin{array}{c}
1 \\
0 \\
\vdots \\
0
\end{array}\right]
$$

Such a choice is clearly stable and satisfies (1b) with $T(z)$ $=A(z)=\operatorname{det} \boldsymbol{H}(z)$. It can be shown (Appendix B) that $\operatorname{det} \boldsymbol{H}(z)$ is all-pass if $\boldsymbol{H}(z)$ is lossless. Hence, the choice (105) ensures that (2) holds with $T(z)$ equal to an all-pass function. In other words, aliasing and amplitude distortion have been completely eliminated.

This observation can be reinterpreted in terms of Fig. 


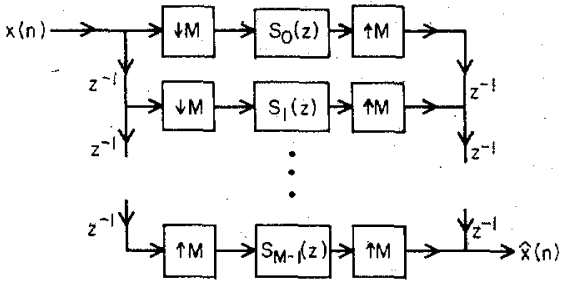

Fig. 24. Pertaining to Appendix A.

10 also. Thus, by property $3.1, \sqrt{M} E(z)$ is lossless when $\boldsymbol{H}(z)$ is lossless. Let us choose $\boldsymbol{R}(z)$ in Fig. 10 according to

$$
\boldsymbol{R}(z)=\operatorname{Adj} \boldsymbol{E}(z)
$$

where

$$
E^{-1}(z)=\frac{\operatorname{Adj} E(z)}{B(z)}
$$

and $B(z)=\operatorname{det} E(z)$. Then

$$
\begin{aligned}
\boldsymbol{P}(z) & =\boldsymbol{R}(z) \boldsymbol{E}(z) \\
& =\boldsymbol{B}(z) \boldsymbol{E}^{-1}(z) \boldsymbol{E}(z)=B(z) \cdot \boldsymbol{I} .
\end{aligned}
$$

With this choice of $\boldsymbol{R}(z)$, Fig. 10 reduces to Fig. 24, where $S_{k}(z)=B(z)$ for all $k$. Thus, by Appendix A, aliasing is absent, and $T(z)=\hat{X}(z) / X(z)=z^{-\langle M-1\rangle}$ $B\left(z^{M}\right)$. Since $\sqrt{M} \boldsymbol{E}(z)$ is lossless, $B(z)$ is again all-pass (except for a scale factor), and thus, amplitude distortion has been eliminated.

In summary, because of the properties of the determinants of lossless matrices, it is possible to eliminate (aliasing and) amplitude distortion in IIR QMF banks, when $\boldsymbol{H}(z)$ [or equivalently $\sqrt{M} \boldsymbol{E}(z)$ ] is lossless. Specific instances of such reconstruction can be found in recent publications [32].

\section{Summary and Conclusions}

In this paper we have considered maximally decimated $M$-channel parallel QMF structures of the form shown in Fig. 1. We showed in Section III how perfect reconstruction can be accomplished by restricting $\boldsymbol{E}(z)$ to be FIR and lossless (i.e., unitary on the unit circle). This is equivalent to forcing the $\mathrm{AC}$ 'matrix $\boldsymbol{H}(z)$ to be FIR and lossless. A lossless AC matrix $\boldsymbol{H}(z)$ implies that the analysis filters $\left\{H_{k}(z)\right\}$ satisfy the "power-complementary" property, i.e.,

$$
\left|H_{0}\left(e^{j \omega}\right)\right|^{2}+\left|H_{1}\left(e^{j \omega}\right)\right|^{2}+\cdots+\left|H_{M-1}\left(e^{j \omega}\right)\right|^{2}=1 .
$$

Conversely, (109) does not imply that $\boldsymbol{H}(z)$ is lossless and is by no means a sufficient condition for perfect reconstructibility. In Section III several properties pertaining to perfect reconstruction were presented and, based on some of these, it was also verified in Section IV that the tree structured SB QMF bank has a lossless AC matrix.

We feel that for $M>2$, the problem of designing $H_{k}(z)$ should not be approached from the point of view of spectral factorization of $M$ th band filters, because such an approach cannot lead to $H_{k}(z)$ with good stopband attenuation if $\boldsymbol{H}(z)$ is at the same time forced to be lossless. The results of Section VI place in evidence one method for obtaining a set of analysis filters which satisfy the sufficient conditions for perfect reconstruction. A procedure has also been presented whereby the coefficients of such an FIR analysis bank can be optimized in order to provide a good stopband attenuation for each of $H_{k}(z)$. The optimization problem in Section VI opens up several possibilities for improving the design algorithm, and some of these are currently under study.

\section{APPENDIX A}

Consider the $M$-channel structure shown in Fig. 24. It is easily, seen that $\hat{X}(z)$ in this figure is given by

$$
\hat{X}(z)=\frac{z^{-(M-1)}}{M} \sum_{l=0}^{M-1} X\left(z W^{l}\right) \sum_{k=0}^{M-1} S_{k}\left(z^{M}\right) W^{-k l} .
$$

- From here we can deduce that the structure is free from aliasing if and only if $S_{k}(z)$ is independent of $k$, i.e.,

$$
S_{k}(z)=S(z) \text { for all } k \text {. }
$$

Under this condition, we have $\hat{X}(z)=z^{-(M-1)} S\left(z^{M}\right)$ $X(z)$. In particular, if $S(z)=1$ then $\hat{X}(z)=z^{-(M-1)}$ $X(z)$, which in turn is also clear by inspection of Fig. 24 . This also provides a simple example of a perfect-reconstruction system.

\section{ApPENDIX B}

Let $\boldsymbol{H}(z)$ be lossless. Then

$$
\boldsymbol{H}_{*}^{T}\left(z^{-1}\right) \boldsymbol{H}(z)=I, \quad \text { for all } z
$$

Taking the determinant of (A.3),

$$
\operatorname{det} \boldsymbol{H}_{*}^{T}\left(z^{-1}\right) \operatorname{det} \boldsymbol{H}(z)=1, \quad \text { for all } z,
$$

i.e.,

$$
A_{*}\left(z^{-1}\right) A(z)=1, \quad \text { for all } z
$$

where $A(z) \triangleq \operatorname{det} \boldsymbol{H}(z)$. This shows that $A(z)$ is allpass.

\section{ACKNOWLEDGMENT}

We wish to acknowledge encouraging and useful discussions we have had during the ICASSP' 86 with R. E. Crochiere of the AT\&T Bell Labs, T. Barnwell, III, of the Georgia Institute of Technology, T. E. Ramstad of the Norwegian Institute of Technology, and $\mathrm{M}$. Vetterli of the Ecole Polytechnique Federale de Lausanne.

\section{REFERENCES}

[1] A. Croisier, D. Esteban, and C. Galand, "Perfect channel splitting by use of interpolation/decimation/tree decomposition techniques," presented at the Int. Conf. Inform. Sci. Syst., Patras, Greece, 1976.

[2] D. Esteban and C. Galand, "Application of quadrature mirror filters to split-band voice coding schemes," in Proc. IEEE Int. Conf. Ac- 
coust., Speech, Signal Processing, Hartford, CT, May 1977, pp. 191195.

[3] R. E. Crochiere and L. R. Rabiner, Multirate Digital Signal Processing. Englewood Cliffs, NJ: Prentice-Hall, 1983.

[4] V. K. Jain and R. E. Crochiere, "Quadrature mirror filter design in the time domain," IEEE Trans. Acoust., Speech, Signal Processing, vol. ASSP-32, pp. 353-361, Apr. 1984.

[5] C. R. Galand and H. J. Nussbaumer, "New quadrature mirror filter structures," IEEE Trans. Acoust, Speech, Signal Processing, vol. ASSP-32, pp. 522-531, June 1984.

[6] M. J. T. Smith and T. P. Barnwell, III, "A procedure for designing exact reconstruction filter banks for tree structured subband coders," in Proc. IEEE Int. Conf. Acoust., Speech, Signal Processing, San Diego, CA, Mar. 1984, pp. 27.1.1-27.1.4.

[7] - "A unifying framework for analysis/synthesis systems based on maximally decimated filter banks," in Proc. IEEE Int. Conf. Acoust., Speech, Signal Processing, Tampa, FL, Mar. 1985, pp. 521-524.

[8] H. J. Nussbaumer, "Pseudo QMF filter bank," IBM Tech. Disc. Bull., vol. 24, no. 6, pp. 3081-3087, Nov. 1981.

[9] P. L. Chu, "Quadrature mirror filter design for an arbitrary number of equal bandwidth channels," IEEE Trans. Acoust., Speech, Signal Processing, vol. ASSP-33, pp. 203-218, Feb. 1985.

[10] J. H. Rothweiler, "Polyphase quadrature filters-A new subband coding technique," in Proc. 1983 IEEE Int. Conf. Acoust., Speech, Signal Processing, pp. 1280-1283, Boston, MA, Mar. 1983.

[11] K. Swaminathan and P. P. Vaidyanathan, "Theory of uniform DFT, parallel quadrature mirror filter banks," presented at the IEEE Int. Conf. Acoust., Speech, Signal Processing, Tokyo, Japan, Apr. 1986.

[12] - , "Design of uniform DFT, parallel FIR quadrature mirror filters," presented at the IEEE Int. Symp. Circuits Syst., San Jose, CA, May 1986.

[13] $\_$, "Theory and design of uniform DFT, parallel quadrature mirror filter banks," IEEE Trans. Circuits Syst., vol. CAS-33, pp. 11701191, Dec. 1986.

[14] M. Vetterli, "Splitting a signal into subsampled channels allowing perfect reconstruction," in Proc. IASTED Conf. Appl. Signal Processing Digital Filtering, Paris, France, June 1985.

[15] _- "Filter banks allowing for perfect reconstruction," Signal Processing, vol. 10, no. 3, pp. 219-244, Apr. 1986.

[16] F. Mintzer, "Filters for distortion-free two-band multirate filter banks," IEEE Trans. Acoust, Speech, Signal Processing, vol. ASSP33, pp. 626-630, June 1985 .

[17] P. P. Vaidyanathan and S. K. Mitra, "Low passband sensitivity dig ital filters: A generalized viewpoint and synthesis procedures, "Proc. IEEE, pp. 404-423, Apr. 1984.

[18] P. P. Vaidyanathan, "New cascaded lattice structures for FIR filters having extremely low coefficient sensitivity," in Proc. IEEE Int. Conf. Acoust., Speech, Signal Processing, Tokyo, Japan, Apr. 1986, pp. 497-500.

[19] - "Implementation of arbitrary FIR transfer functions as passive cascaded lattice structures," presented at the IEEE Int. Symp. Circuits Syst., San Jose, CA, May 1986.

[20] — "Passive cascaded lattice structures for low sensitivity FIR filter design, with applications to filter banks, "IEEE Trans. Circuits Syst., vol. CAS-33, pp. 1045-1064, Nov. 1986.

[21] F. Mintzer, "On half-hand, third-band, and $N$ th-band FIR filters and their design," IEEE Trans. Acoust., Speech, Signal Processing, vol. ASSP-30, pp. 734-738, Oct. 1982

[22] V. Belevitch, Classical Network Synthesis. San Francisco, CA: Holden-Day, 1968.

[23] B. D. O. Anderson and S. Vongpanitlerd, Network Analysis and Synthesis. Englewood Cliffs, NJ: Prentice-Hall, 1973.
[24] A. Fettweis, "Pseudopassivity, sensitivity, and stability of wave digital filters," IEEE Trans. Circuit Theory, vol. CT-19, pp. 668-673, Nov. 1972.

[25] T. Kailath, Linear Systems: Englewood Cliffs, NJ: Prentice-Hall, 1980.

[26] F. D. Murnaghan, The Unitary and Rotation Groups. Washington, DC: Spartan Books, 1962

[27] The IMSL Library, A set of Fortran subroutines for mathematics and statistics.

[28] P. E. Gill, W. Murray, and M. H. Wright, Practical Optimization New York: Academic, 1981.

129] P. P. Vaidyanathan, "On power-complementary FIR filters," IEEE Trans. Circuits Syst., vol, CAS-32, pp. 1308-1310, Dec. 1985.

[30] M. G. Bellanger, G. Bonnerot, and M. Coudreuse, "Digital filtering by polyphase network: Application to sample-rate alteration and filter banks," IEEE Trans. Acoust., Speech, Signal Processing, vol. ASSP24, pp. 109-114, Apr. 1976.

[31] A. G. Constantinides and R. A. Valenzuela, "An efficient and modular transmultiplexer design," IEEE Trans. Commun., vol. COM-30 pp. 1629-1641, July 1982 .

[32] A. Fettweis, J. A. Nossek, and K. Meerkotter, "Reconstruction of signals after filtering and sampling rate reduction," IEEE Trans. Acoust., Speech, Signal Processing, vol. ASSP-33, pp. 893-902, Aug. 1985.

[33] T. H. Ramstad, "Analysis/synthesis filter banks with critical sampling," presented at the Int. Conf. Digital Signal Processing, Florence, Italy, Sept. 1984.

[34] T. P. Barnwell, III, "Subband coder design incorporating recursive quadrature filters and optimum ADPCM coders," IEEE Trans. Acoust., Speech, Signal Processing., vol. ASSP-30, pp. 751-765, Oct. 1982.

[35] G. Wackersreuther, "Some new aspects of filters for filter banks," IEEE Trans. Acoust., Speech, Signal Processing, vol. 34, pp. I1821200 , Oct. 1986.

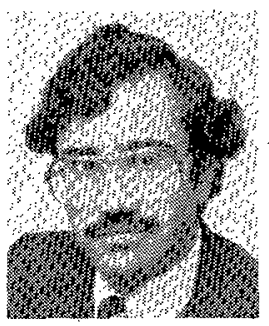

P. P. Vaidyanathan ( $\mathrm{S}^{\prime} 80-\mathrm{M}^{\prime} 83$ ) was born in Calcutta, India, on October 16,1954 . He received the B.Sc. (Hons.) degree in physics, and the B. Tech. and $\mathbf{M}$. Tech. degrees in radiophysics and electronics from the University of Calcutta, India, in 1974, 1977, and 1979, respectively, and the $\mathrm{Ph} . \mathrm{D}$. degree in electrical and computer engineering from the University of California, Santa Barbara, in 1982 .

He was a Postdoctoral Fellow at the University of California, Santa Barbara, from September 1982 to February 1983. Since March 1983 he has been with the California Institute of Technology, Pasadena, as an Assistant Professor of Electrical Engineering. His main research interests are in digital signal processing, linear systems, and filter design.

Dr. Vaidyanathan served as the Vice Chairman of the Technical Program Committee for the 1983 IEEE International Symposium on Circuits and Systems. He currently serves as an Associate Editor for the IEEE TRANSACTONS ON CIRCUITS AND Systems. He was the recipient of the Award for Excellence in Teaching at the California Institute of Technology for 1983-1984. He was also a recipient of the National Science Foundation's Presidential Young Investigator Award in 1986. 